

\section{Bot.Dict

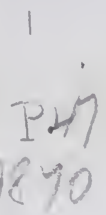

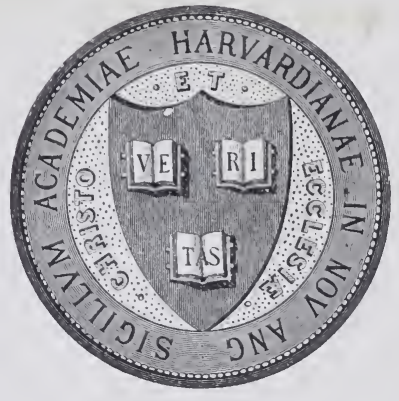

I I IR A R Y

()F THE

(isay Herbarium.

26 Fel. 1896. 
Digitized by the Internet Archive in 2017 with funding from BHL-SIL-FEDLINK

https://archive.org/details/synonymiaebotani00pfei_0 


\title{
SYNONYMIAE BOTANICAE
}

\author{
1870 editae
}

\section{Supplementum primum.}

\author{
CURAVIT
}

Dr. Ludovicus Pfeiffer

Cassellanus.

CASSELLIS.

Sumptibus Theodori Fischeri.

1874. 


\title{
Synonymik
}

\section{der botanischen Klassen-, Familien-,}

\section{Gattungs- und Sectionsnamen.}

\author{
Erstes Supplement
}

zu dem 1870 erschientenen Werke

von

\section{Dr. L. Pfeiffer \\ in Cassel.}

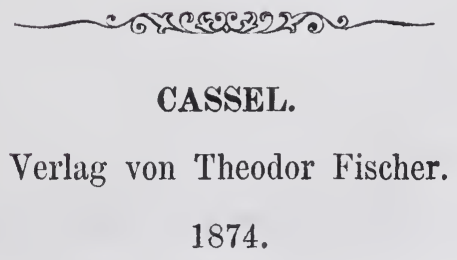




\section{Druck von Döll \& Schäffer (L. Döll) in Cassel.}




\section{Praemonenda.}

Ecce primum Synonymiae botanicae, 1870 a me editae, supplementum, sat magnum synonymorum et nominum novorum in illo opere ob deficientem librorum studii occasionem praetermissorum numerum continens.

Steudelii Nomenclator (ed. secunda 1840. 41), quem antea ob typographicorum aliorumque errorum molem fere omnino consulto praeteriveram, suadentibus amicis nunc receptus est et quasi truncum Supplementi nostri suppeditavit; praeterea synonyma (et sphalmata graviora) in Dumortieri scriptis 1829 et 1835 , auctoris benevolentia nuperius mihi communicatis, in Wightii Iconibus plant. Indiae (1838-1852), in Dietrichi Synopsi (1839-1843) occurrentia, nec non nomina in Wittsteini Lexico etymologico correcta vel mutata locis suis inserta sunt.

Nomina multa Rumphiana, Plumieriana, Browneana aliorumque, ab auctoribus diverse et plerumque falso citata, studio exacto librorum ipsorum nunc correcta sunt, eoden modo a Ruiz et Pavon, Cavanilles, Jacquin etc. edita jam in "Synonymia" correcta sunt. (Conf. Palaua Ruiz \& Pav., Palaua Cavan., et multa alia.)

Sed aliorum nominum, pro generibus vel sectionibus novis in operibus recentioribus usque ad finem anni 1858 promulgatorum et a me (interdum propria culpa) praetervisorum, imo e scriptis nonnullis $\mathrm{Cl}$. Blume, Miquel, Grisebach, post impressionem Synonymiae excerptorum et in "Nomenclatore botanico" (cujus impressio ad duas tertias jam absoluta est) recensitorum copiam in hoc Supplemento adjeci - et indulgentiam Eorum spero, qui difficultates innumeras ejusmodi laboris noverunt. 
E "generum dubiae sedis vel non satis notorum" copia (n. 12629-12793) multa nunc ad loca apta translata sunt; quae restant et quae satis numerosa in hoc Supplemento accesserunt, examini critico botanicorum peritiorum commendare velim.

Sphalmata in Synonymia occurrentia tandem colrexi et errores nonnullos emendavi, ut liber meus, etsi usui diario minus commodus quam antea, tamen omnia offerat, quae mihi ipsi colligere contigit.

Supplementa ulteriora, annos inde ab 1858 complectentia, Deo favente conjunctis viribus edenda spero.

\section{Dr. L. Pfeiffer.}

\section{Vorwort.}

Als ich bei den Vorarbeiten zu meinem Nomenclator botanicus und gleichzeitig zur Synonymia zum ersten Male die zweite Ausgabe des Steudel'schei Nomenclators (1840. 1841, die erste war mir nicht zugänglich) in Händen hatte und die betreffenden Artikel aus dem Buchstaben $\mathbf{A}$ excerpirt und eingetragen hatte, wurde, es mir zweifelhaft, ob es sich der Mühe lohnen und für die, welche mein Buch benutzen wollten, von hinreichendem Interesse sein würde, wenn ich die Sammlung von Druckfehlern, welche den Gebrauch jenes Werkes als Quelle sehr bedenklich machen, vollständig einregistriren wollte, und ich legte deshalb das Buch zur Seite. Da ich jedoch später fand, dass das Steudelsche Werk vielfach verbreitet war, und mir auch von freundlicher Seite dazu gerathen wurde, so habe ich, nachdem meine Synonymie bereits dem Publicum übergeben und einige Bogen des Nomenclator gedruckt waren, für letzteres Werk die sämmtlichen Steudelschen Varianten noch einrangirt, welche zwar grossentheils nur auf un- 
zweifelhaften leicht zu berichtigenden Druckfehlern beruhen, aber auch zum Theil absichtlichen Veränderungen in der Schreibart ihren Ursprung zu verdanken scheinen, und endlich theilweise (scheinbar nothwendige) neu gebildete Namen für solche Gattungen sind, welche mit schon früher gebrauchten collidirten. Dahin gehören z. B. Baziasa, Beriesa, Ifdregea, Hugelroea, Kennedynella, Trigonocarpaea, Vasargia etc., welche in meiner Synonymik fehlen, aber fast ausnahmslos sich als überflüssig erwiesen haben und nur die Masse der Synonymen bekannter Gattungen vermehren. Freilich konnte und musste ich nun auch nach den Steudelschen Angaben eine Anzahl von Artikeln aufnehmen, welche mir unbekannt geblieben waren und für welche ich auch jetzt die Quellen nicht mehr habe auffinden können und nicht für die Correctheit bürgen kann.

Aehnlich ist es mir mit den abweichenden Namen in Dietrich's Synopsis ergangen, deren Uebergehung mir ebenfalls anfänglich rathsam, später aber doch bedenklich erschien. Ein drittes Werk, dessen Varianten ich wie jene noch in meinem Nomenclator eingeordnet habe, ist W it tstein's Botan. etymol. Handwörterbuch. Diese beruhen, der Tendenz des Werkes entsprechend, grösstentheils auf absichtlichen Veränderungen (gerade nicht immer nothwendigen Verbesserungen) der Schreibart, gestützt auf zum Theil weit gesuchte, zum Theil den directen Angaben der Autoren widersprechende Etymologieen, und mussten nun, meinem Plane entsprechend, ebenfalls aufgenommen werden.

Die Artikel aus diesen 3 Werken, sowie auch aus 2 erst während des Druckes des Nomenclator durch die Güte des Verfassers mir zugänglich gewordenen Werken von Dumortier bilden nun den ganz ansehnlichen Stamm (Std. für Steudel, Wst. für Wittstein und Dtr. für Dietrich) des vorliegenden ersten Supplements zu meiner Synonymia botanica, welches zwar noch nicht über den von Anfang bestimmten Zeitpunkt (Ende 1858) hinausschreiten konnte, aber doch noch eine beträchtliche Anzahl von Artikeln bietet, welche aus Werken, die mir erst in 


\section{VIII}

den letzten Jahren zugänglich wurden, entlehnt sind. Dass darunter mehre wichtige Arbeiten von Blume, Miquel, Grisebach u. A. befindlich sind, muss ich mir selbst vorwerfen, hoffe jedoch Entschuldigung bei Denen zu finden, welche einigermassen die Schwierigkeiten meines Unternehmens zu würdigen wissen. So fand ich ferner durch Eingehen auf die Quellen, dass viele Namen von $R u m p h$, Plumier, P. Browne u. A. fast durchgängig falsch eitirt werden, und diese Berichtigungen finden sich ebenfalls in diesem Supplement, während die ebenfalls vielfach entstellten Namen von Ruiz \& Pavon, Cavanilles u. A. schon in der Synonymie berichtigt sind. Einen nicht unansehnlichen Beitrag lieferten ferner das Studium von B u rmann's Thesaurus zeylanicus, und die zahlreichen Namenvarianten in Wight Icon. pl. Ind., welche zwar wohl grossentheils, wie aus der Bezeichnung der Namen auf den Tafeln hervorgeht, aus flüchtiger oder mangelhafter Correctur hervorgehen, zum Theil aber (auch abgesehen von den neuen Gattungen) absichtlich sein mögen.

Aus der Rubrik "Genera dubiae sedis vel non satis nota" (v. 12629-12793) ist es mir noch gelungen, viele an ihrem richtigen Platze einzuordnen; auf die noch übrig bleibenden, sowie auf die nicht unbeträchtliche Anzahl der in diesem Supplemente hinzugekommenen möchte ich vorzugsweise die Aufmerksamkeit der gelehrten Botaniker lenken, da doch gewiss die meisten derselben mit Gewissheit oder Wahrscheinlichkeit festgestellt werden können.

Hätte ich übrigens alle irgend erreichbaren Quellen vor der Publication selbst prüfen wollen, so hätten weitere Jahrzehende kaum hingereicht. Es wird auch wohl jetzt noch Manches übersehen sein, und ich werde es dankbar anerkennen, wenn ich, wie schon von mehren Seiten freundlich geschehen, auf Mängel aufmerksam gemacht werde, deren Beseitigung noch theils dem Nomenclator, theils späteren Supplementen zu Gute kommen kann!

Cassel, im Januar 1874.

Dr. L. Pfeiffer. 


\title{
Synonymiae botanicae
}

\author{
Supplementum primum.
}

\section{Addenda et corrigenda in conspectu systematico.}

\section{Algae.}

n. 9 add. Fragillaria Math.

- 18 - Discoplaca Breb.

- 51 - Achnanthella Gaill.

- 55 - Syncyctia Breb.

- 59 - Paltonophora Wst.

- 74 - Rhaphidogloca Breb.

- 75 - Homaeocladia Harv.

- 78 - Chalcothrix Breb.

- 79 - Dickica Breb.

- 79/1. Endosigma Breb.

- 97 leg. Endictya Ehrenb. Eudictya Kütz.

- 116 - Chaetoceros Ehrenb. Chaetoceras Kütz.

- 167 add. Evastrum Math. Strephocystis ...?

- 179 - Tessararthra Ehrenb.

- 180 - Sphondylotium Breb.

- 181 - Didymoprion Mont.

- 182 - Desmidia Breb.

- 187 - Selenaca Liudl.

- 189 - Ćrucigenieila Giaill.

- 194/1 ? Arthrotoma Corda.

- 197 add. Aërophyton Wst.

- 212 - Ascidium Braun.

- 219 - Aneucystis Breb.

- 253/1 ? Helminthonema Kütz.

- 264 leg. Volvox Müll.

- 272 vid. 423.

- 290 leg. Iueibleinia M e n egh.

- 291 add. Lyngbia Math.

- 295 - Anabainella Gaill.

- 301 - Nostochia Duby.

- 305 - ? Percursaria Bonnem.

- 317 - Dictionema Men.

- :318/1. Zoogloea Cohn.

- 329 add. Gloeotrichia Mont.

- 343 - Draparnalia Ritg.

- 345 - Diadenia Wst.

- 353 - Lamourouxella Bory pt. n. 355 ald. Psichormium Mont.

- 377 - Audouinella Bonnem.

- 386 - Spirogera Dumort.

- 389a leg. 'Tendaridea Bory. Tyudaridea Rchb. Tentaridea Math.

- 392 add. Botrytella Bory.

- 397 leg. Sphacellaria Bory.

- 400 add. Dasytricha Wst.

- 413 - Ilaea Dumort.

- 423 leg. Ophiocytium Näg. Spirodiscus Eichw. Brochidium Perty. Ophiothrix Kütz.

- 437 add. Rhipidium Wst.

- 438 - Avrainvillaea Lindl.

- 439 - Opuntia Nacc. Sertolara Nardo.

- 443 - ? Physydrum Raf.

- 452 - Polyphisa Lindl.

- 458 - Talerodictyon Lindl.

- 460a - Eunitella Braun.

- 461a - Euchara Braun.

- 471 - ? Batrachosperma Bory. BatrachospermellaGaill.

- 479 - Leathina Lindl.

- 491 - Spilophora Wst.

- 514 - Desmaretia Dumort.

- 516 - Phloeorrhiza Mont.

- 520 - Haligeria Lindl.

- 539 - Hymanthalia Dumort.

- \$40 - Myriadena Mont.

- 546 - Ozothalia Lindl.

- 554 - Cystoscira Math.

- 557 - Halydrys Harv.

- 572 - Blosvillea Decne.

- 577 leg. Scabeira M e n e gh.

- 580 add. Callithammion J. Ag.

- 58.3 - Sirospora Mont.

- 587 - Halodictyon Mont.

- 592 leg. Spirydia M enegh.

- 597 add. Hanovia Hont. 
n.622 leg. Peysson ellia Decne. Peyssonelia Kütz. Peysonnellia Lindl.

- 623 - Hildbrandtia Nardo. Hildenbrandtia $\mathrm{R}$ ch $\mathrm{b}$. Hildenbrantia Nen.etc.

- 627 add. Pneophilum Mont.

- 628 - Aghardina Nardo.

- 630 - Cellipora Linn.

- 631b - Eurytium Wst.

- 632 - Nodolara Imper. Titanephlium Nardo. Titanephyllum Endl. Haliptilon Lindl.

- 635 - Dudresneya Wst.

- 658 - Kallymena Wst.

- 673 leg. Acantholobus Kütz. Acanthobolus Ej.

- 681 - Hypnaea Bory.

- 720 add. Trichothamnium Breb.

- 723 - Hutchintia Bory. Syennema Spr. pt. Grammalia Dumort.

- 729 - Bonnemaisonuia Mont.

- 731 leg. Rhytiphlaea Fries.

- 735 add. Spirrymenia Mont.

- 737 - Epineurron Math.

- 758 - Pollexfexia Lindl.

- 790/1. Pilobolus A. Braun.

- 808/3 ? Pectoralina Bory.

- 808/4. Pemphidia Dumort.

- 812/1 vid. 723.

- 820/1. Corallinites Ung.

- 822 add. Cystosirites Mont.

- 831 leg. Haliserites Sternb. Halyserites Ung.

- 834 add. Munsteria Brgn.

- 834/1. Phymatoderma Brgu.

\section{Lichenes.}

n. $863 / 1$ ? Pasithoë Decne.

- 886 add. ? Stephanophorus Flot.

- 887 leg. Micaraea Mont.

- 920 add. Limbaria Wst.

- 936 - Caliciois Math.

- 939 - Conyocybe Math.

- 949 leg. Arthronia Du m ort. add. Ardonia Mont.

- 950 - Coniocarpum Wst.

- 958 - a. Sphaeralidia Fée. b. Serialidia Ej.

- 962 leg. Tripethelium lumort. add. a. 'I'ripethelina Fée. b. Amorphalia Ej.

- 963 - Porodothium Wst.

- 978 - Patellastrum Mont. n.1006 add. Rhizocarpum Wst.

- 1012 - Rhaphiospora Theob.

- 1058 - Omphalidium Lindl.

- 1062 - Plectocarpum Wst.

- 1067/1 ? Caenogium Lindl.

- 1079 leg. ('hlorea Nyl.

- 1091 add. Roccelia Dumort.

\section{Fungi.}

n.1142 add. Phyllirium Math.

- 1189/1. Trichobasis Lev.

- 1193 add. Coniosporum Wst.

- 1205 add. Oevidium Lev.

- 1212 - Aptenoum Lev.

- 1232 - Botryospora Lindl.

- 1244 - Clasterispora Lindl. Clasterosporium Lev.

- 1245 - Ceratospora Lindl.

- 1252 - Soredosporium Lindl.

- 1256 - Speirea Lev.

- 1279 lg. Mydonotrichum Cord a. Midonotrichum Lev.

- 1288 add. Caelosporium Lindl.

- 1292 - Midonosporium Lev.

- 1294 - ? Mitrosporium Lev.

- 1302 - Azozma Lindl.

- 1304 - Echinobotrys Lindl. Sporodon Lev.

-1305 - Cystopora Lev.

- 1313 - Mainomyces Lev.

- 1314 - Aleurhisma Lev.

- 1324 - Plecotricum Lindl.

- 1338 leg. Crinotrichium Ritg.

- 1353 add. Styzanus Mont.

- 1360 - Gonatorhodius Lindl. Gonatorrhodon Lev.

- 1365 - Spondycladium Lev.

- 1369 - Hapalaria Dumort.

- 1384 - Strachybotrys Lev.

- 1390/1 ? Pterodinia Chev.

- 1390/2 ? Streblocaulium Chev.

- 1391 add. Tricothecium Mont.

- 1401 - Pteronospora Lindl.

- 1404 - CephalosporumLindl.

- 1406 - Chlonostachys Lev.

- 1407 - Choretopsis Lev.

- 1416 - Theleactis Lev.

- $1420^{\circ}$ - Syzigites Lindl. Sysygites Mont.

- 1430 - Chionypha Lev.

- 1438 - Sphaerospora Lindl.

- 1448 - Crocisporium Lev.

- 1450 - Myxomphalon Lindl.

- 1454 - Iisporium Leman.

- 1458 - Rhizoctonum Leman.

- 1465 - Solenosporium Lev. 
n.1472 add. Trimmatospora Lev.

- 1508 - Calycosporium Lev.

- 1513c - Cilicipodium Lev.

- 1518 - Gloiocladium Lev.

- 1525 - Amphicorda Lindl.

- 1538 - Trichostoma Wst.

- 1559 - Tricamphora Lev.

- 1564c - Pseudocormus Wst.

- 1565 - Polychysmium Lev.

- 1568 - Angionidium Ler.

- 1575 - Ptycogaster Lev.

- 1580 - Galependrum Leman.

- 1581 - Mysothecium Iindl.

- 1591 - Onigena Math.

- 1599 - Hypochnaena Wst.

- 1608 - Steerbeckia Dumort.

- 1609 - Scoleiocarpus Lev.

- 1616 - Endoneuron Lev.

- 1625e - Actinodermum Lev.

- 1627 - Astricium Lev.

- 1629/1 ? Riella Rafin.

- 1629/2 ? Suspicante Schw.

- 1642 add. Cyathea Lev.

- 1661 - Pachyphlaeus Lindl.

- 1670 - Bulliardia Wst.

- 1672 - Gauthiera Lev.

- 1673/1 ? Bromicolla Eichw.

- 1675 c leg. Cletria P. Browne. Clethria Fries.

- 1678 - Mo-ku-sin Cibot.

- 1683 add. Dictyopeplus Wst.

- 1685/1 ? Atractus Kirby.

- 1687 add. Xilaria Math.

- 1700 - Pyrenochium link.

- 1702 - Dumortieria West.

- 1702c - Cyathosphaera Dum.

- 1702g - Siphosphaera Ej.

- 1702k - Stigmasphaera Ej.

- 1702m - Thallosphaera Fj.

- 1702t - Mitrosphera Ej.

- 1707 - Aylographa Math.

- 1708b - Scopinella Lev.

- 1714 - Pemphydium Lev.

- 1724/1. Synalissis Lindl.

- 1729/1. Aposphaeria Berk.

- 1740 leg. P h lo e o scoria Wallr.

- 1749 add. Podostromium Lindl.

- 1750 leg. Erysibe Theophr., Wallr. (Non Murr., nec Rebent).

- 1766 add. Couturia Lindl.

- 1768 - Clissosporium Lev.

- 1798 - a. Macroplodia West. b. Diplodina Ej.

- 1805 - Dilophosporium Lindl. n.1807 leg. Colletothrichum Ej. - 1809/1 ? Spilobolus Link, I,ev.

- 1829 add. Phlaeospora Westend.

? b. Rhabdospora Ej.

- 1836 - Melittiosporium Lindl. Melittosporium Lev.

- 1838 leg. Xylogramma Wallr.

- 1839/1 ? Godronia Moug. et Lev.

- 1856a add. Histerium DC.

- 1870b - Sclerodermis Lindl.

- 1905 - ? Petellaria Dumort.

- 1905d leg.Hymenos c yphus Nees.

- 1905m $\gamma$ - Acetabularium Ej.

- 1905/2. Desmazierella Lib.

- 1915 add. Mitrularia Dumort.

- 1919 - Fromitra Lev.

- 1931 - Typhella Math.

- 1935 - Clavariastrum Math.

- 1948/1 ? Hypolyssus Berk.

- 1951 del. Hygromitra Fries.

- $1952 \mathrm{~g}$ add. Jachnocladium Lev.

- 1954/1. Leptochaete Lev.

- 1958 add. Richnophora Leman.

- 1965 - Hypnois Math.

- 1971 - Glaeoporus Lindl.

- 1977 - Vonkhout Wst.

- 1978 - Junguhnia Lev.

- 1987 - Schyzophyllum Dum.

- 1994 - Lentiscyphi Fries.

- 1995 - Marasmium Wst.

- 1998 - Cantharulus Beckm. Leptopilos Duby.

- 2008p - Fccilea Lev.

- 2008y - Clytocybe Lindl.

- 2008aa - Calantica Lev.

- 2014/1. Acrophyton Eschw.

- 2037/1. Malacharia Fee.

- 2039/1. Mediastina Dod.

- 2041/1. Mitrosporium Corda.

- 2043 add. Myxacium Lev.

- 2048 - Phyllaedium Lindl.

\section{Hepaticae.}

n.2064 add. Hemiseurnata Lindl.

- 2065 - Tessellina Dumort.

- 2069 leg. Duri a e a Bory \&Mont.

- 2071 - Carpoc er os Dumort. add. Ceranthus Linn.

- 2076 - Racotheca Wst

- 2080 - Raciocarpon Wst.

- 2081 - Nemoursia Merat.

- 2085 - Marcantia P. Br.

- 2092 - b. Acrostolia Dumort.

- 2095 leg. Rhizophyllum Pa lis.

- 2096 - Hymenophyton Dumort. 
n.2096 add. Symphyogyna Nees. Symphyogyne $W_{\text {st. }}$.

- 2108 - Physananthus Lindl.

- 2113 leg. Lepidolaena Dumort. Polyotus Gottsche.

- 2114b add. Lepicolea Dumort.

- 2115 leg. Thricolea 1)umort.

2116 Tricholea D un ort

- 2116 - Pleurozia Dumort. Physiotium Nees.

- 2119 - Pleuroschisma Dum. Mastigobryum Nees.

- 2122 add. Calypogea Dumort.

- 2125 - Acrobolbos Lindl.

- 2127 - Mursupella Dumort.

- 2134 leg. Odontoschisma Dum. Sphagnoecetis Nees.

- 2135 add. Cephalozya Wst.

- 2137 - Carpolepidium Leman.

- 2139 leg. Schistochila Dumort. Gottschea Nees.

- 2140 add. Mosophylla Dumort.

- 2143 - Mniopis Dumort.

\section{Musci.}

n.2150 add. Andreoer Mont.

- 2159 - Schistotega Dumort.

- 2164 - Skytophyllum Wst.

- 2178 - Cryptodon R. Brown.

- 2181a - Systiliun Mont.

- 2182 - Haplodon Mont.

- 2184 - Saccophorum Wist.

- 2185 - Cinclidum Dumort.

- 2186b - Peromnium Mont. Streptotheca Arn.

- 2188 - Tetraphys Mont.

- 2193 - Atrichium Wst.

- 2194 - g. Lipotrichum Mont.

- 2195 - Triphocoma Wst.

- 2197 - Mielighoferia Wst. Schizymenium Miont.

- 2200 - Bryastrum Duby.

- 22001) - Amphirrhinum Wist.

- 2206d - Thesanomitrion Math.

- 2209 - ('ampylostylium Mont.

- 2214 - Ditrichum Wst.

- 2218 leg. Tristichitis Ehrh. 'Tristichis Wst.

- 2225 - Eucalypta Dumort. ? Extinctorium Ej.

- 222Sd add. Armnoweissia Mont.

- 2235 - Weissa Schrank.

- $2235 \mathrm{~b}$ - Weissiopsis Mont.

- 2239b - Nauia Borkh.

- 2245 - Ptychomitrion Mont.

- 2259c - Pterogenium Angstr. n.2260 add. Pilotricum Palis.

- (2260c) Meteorium Brid.

- 2260h leg.Pterobryon Horn sch.

Ptirobryum C. Müll.

Cryptotheca Hornsch.

- $2265 \mathrm{c}$ add. Homalia Wst.

- 2270/1. Herpodon Müll.

- 2270/2. Microbryum Br. \& Sch.

- 2270/3. Aplotrichum Dumort.

\section{Calamariae.}

n.2771 add. Equisitum P. Browne.

- 2282 leg. Bruk mannia Sternb. Bruckmanuia Nonnull.

- 2282/1. Calamodendron Brgn.

\section{Filices.}

n 2286 add. Nevropteris Brgn.

- 2286/1. Nephropteris Brgn.

- 2289/1. Pychnophyllum Brgn.

- 2291 add. Linopteris Sternb.

- 2293 leg. Sphaenopteris S ter nb. add. Sphenopecopteris Stb.

[a. Davallioides Ung.

b. Cheilanthoides Ej.

c. Dicksonioides Ej.]

- 2298 add. Desmophlebis Brgn.

- 2301/1. Hausmannia Dunk.

- 2304 add. Dictyopteris Brgn.

- 2305 - Campylopteris Strnb.

- 2306 - Guttbiera Brgn.

- 2312 - a. Notopteris Presl. b. Acropteris Ej.

- 2312/1. Goniopterites Brgn.

- 2313 add. Apocynophyllum Vis. \& II.

- 2313/1. Phyllopteris Brgn.

- 2314 add. a. Eupecopteris Strnb. b. Orthopleuria Ej.

[a. Aplophlebis Brgn. b. Dicrophlebis Ej.]

- 2314/1. Cladophlebis Brgn.

- 2314/2. Coniopteris Brgn.

- 2:61 add. Lomoria Dumort.

- 2370 - Vitteria Reuss.

Haplopteris Presl.

- 2372 - Chilogramma Wst.

- 2380 - Diclidopteris Brack.

- 2382 - AmphradeniumBrgn.

- 2402 - Litholorochia Brgn.

- 2423 - Antrophium Brgn.

- 2437 - Stenogramma Brgn.

- 2445 - Diplasium Wst.

- 2454 - Calymnodon Brgn.

- 2469 - Microsorus Wst.

- 2478 leg. Amblya B rgn. 
Filices, Hydropterides, Selagines, Zamiae, Rhizanthae, Glumaceae. 5

n.2486 add. Athyrium Math.

- 2487 - Aspidium Math.

- 2491 - Mephrodium Dumort.

- 2499 - Fadgenia Lindl.

- 2513 - Hymenostomia Wst.

- 2526 - Cystidium Lindl.

- 2529 - Arachnoides Wst.

- 2532 leg. Hemithelia $\mathrm{Brg} n$. add. Hemitalia Dumort.

- 2532b - Disphaenia Brgn.

- 2536/2 ? Schizochlaena Smith.

- 2544 add.Laccopteris Pr..Sternb.

- 2545 leg. Laccopteris Göp

- 2546 add. Andriana Brgn.

- 2556 - Schizea Brgn.

- 2584 - Helmintostachys Brg.

- 2587 vid. 2380.

- 2588 - 2370.

- 2591/1. Staphylopteris Presl.

- 2594 add. Endogenites Spr. pt.

- 2600 - Tempskia Brgn.

- 2605 - Ptilorachis Brgn.

VIII. Hydropterides.

n.2612 leg. Narsilaea Du mort.

\section{Selagines.}

n.2633 add. [a. Lepidota Sternb. b. Alveolaria Ej. c. Variolaria Ej. d. C'alamites Ej.l

- 2636 leg. Negaphytum Art., Stb. Megaphyton Endl.

\section{Zamiae.}

n.2653 add. Todda-panna Rheede.

- 2654 - Encephalartus Std.

- 2659/1 ? Dipsacozamia Lehm.

- 2664b leg. Nilsonia Brgu.

- 2666 add. Cycadoidea Brgn

- 2 fi67/1. Colpoxylon Brgn.

- 2668 add. Calamoxylum Brgn.

\section{Rhizanthae.}

n 2675 add. Ichtyosina Std.

- 2680 - Sarcocordylis Wall.

- 2684 - Haematostrobos Wst.

- 2687/1. Sarna Karst.

- 2690 add. Brugmannsia Std.

\section{Glumaceae.}

[A. Graminea e.] n.2699 add. Malteburnia Std.

- 2711 leg. Leptaspis R. Brown.

- 2714 add. Linospartum Std. n.2715 add. Thalysia Linn.

- 2717 - Lachryma Jobi Burm.

- 2721 - Cornucopieae Dum.

- 2722 - Pechea Pourr.

- 2729 - Bruckmannia Wst.

- 2732 - Iasiotrichos Lehur.

- 2733 - Symbasiandra Willd.

- 2744 - Depretzia Dtr.

- 2748 - Cleachue Rol. Axopus Dumort. Axinopus (Endl.) Wst.

- 2756 - Trasya Dtr.

- 2761 - Aulaxis Std.

Paractaemum Dum. Sphaerocaryum Nees. Monachme Dumort. Mönchia Wend. Steinschisma Raf., Endl.

Ptychophyllum A. Braun.

- $276 t^{\circ}$ - Menilis Dumort. Nelinus Dtr.

- 2768b - OrthopogonA.Spreng.

- 2770/1. Amphochaeta Anders.

- 2771b add. Gymnostrix Dtr. Cataterophora Std.

- 2771/1. Beckeropsis Fig.\& Not. Beckera Nees.

- 2771/2. Eriochaeta Fig. \& Not.

- 2782 add. Ixalum Forst.

- 2787 - Centosteca. Isis.

- 2810 - Trichosantha Std

- 2818 - Muhlembergia Dum. Dactylograma Link. Serigrostis Std.

- 2822 - Lygurus Dtr.

- 2824 - Phipsia Wst.

- 2829 del. Bennetia Rafin.

- 2830 add. Bennetia Rafin.

- 2833 - Nowodworskia Std. Chaetotropsis Dtr.

- 2837 - Atherophora Willd.

- 2839 - Pteropodium Willd.

- 2849 - Graphophorum Dtr.

- 2850 - Czernia Stil. Phragmitis Beck.

- 2859b - Polyrrhaphis Wst.

- 2861 leg. Cottea Kunth. Cottaea Endl.

- 2864 add. Catestecum Dtr. Catasetum Ej.

- 2866 - Schönfeldia Wst.

- 2868 - Dactylotenium Dum.

- 2870 - Codonachne W. \& A. Phacellaria Willd. 
n.2880 add. Antichloa Sweet. Boutelouoa Wst.

- 2882 - Tristania Poir.

- 2883 - Eutriania Dtr. Nestlera Willd.

- 2888 - Strombodurus Will.

- 2892 - Pleurophus D)tr.

- 2895/1. Casiostega Rupr.

- 2906d add. Acrospelton Wst.

- 2906/1. Monandraira Desv.

- 2908 add. ? Helicotrichum Bess. Helicmotrichum Std.

- 2915 - Trichopterys Wst.

- 2919 - d?Centropodia R.Brown.

- 2919/1. Trichoneura Anders.

- 2923 add. Dasyorhloa Willd.

- 2923a - Diplocoea Std.

- 2924 - Trioidia Dtr.

- 2925 - Rovlea Nees.

- 2929 - ? Plotia Schreb. ? Desmazeria Dum.

- 2932 - Erochloa Std.

- 2937a - Festucaria Fabr.

- 2941 - Lepitoma Torr.

- 2945 - Neuroloma Std.

- 2946 - Chrascolytrum Isis. Chascolythrum Std.

- 2947 - Calosteca Isis.

- 2961a - Phalona Std.

- 2962 - Tinaea Garz.

- 2964 - Lophatherium Wst.

- 2965 leg. Elythrophorus Dum.

- 2966 add. Plagioelytrum Lindl.

- 2967a - ? Megalurus Wst.

- 2967b - Schoenodoros Wst.

- 2968 - Schlerochloa Parl.

- 2970 - Scleropoa Dum., Gris.

- 2992 - Merostachis Std.

- 2994 - StematospermumWst.

- 3000 - Besha Dtr.

- 3002/1 ? Bambusoides Wall.

- 3003 add. Crepalia Schrk, Std.

- 3006 - Elytregia Isis.

- 3008 leg. Nardurus R chb.

- 3018a add. Zeocrithum Beckm. Zeopyrum Trin.

- 3018b - Crithesium Wst.

- 3034 leg. Ophiuros Gärtn. f. Ophiurus R. Brown. Ophyurus Dtr.

- 3035 add. Sanguinella Gleich.

- 3042 - Thyridostachium Wst.

- 3043 - Rottbölia Dumort.

- 3043c leg. Coelorachis Brgn. Coelorhachis Endl. Coelorrhachis Wst. n.3050 add. Leptotherium Dtr.

- 3054 - ? Pterigostachyum Nees.

- 3056b - Eudimeria Lemr.

- 3060 - ? Plazeria Willd., Std.

- 3065 - Pogonantherum Decne. b. Diplatherum Brgn.

- 3066 - Rhipidium Wst.

- 3072 - Anthristiria Dumort.

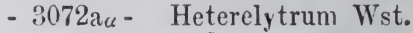

- 3084 - Andrapogon Roy. Andropogum Brgn. Callichloë Willd. Vetiveria Virey.

- 3085 - a. Distachya Brgn. b. Polystachia F.j.

- 3088 - Telopogon Dtr.

- 3091 - AlloterrhopsisA.Rich.

- 3093 - Homoeantherum Std.

- 3096 leg. Athenanthia Kunth. Athenantia Dumort.

- 3096/1. Arthrochloa R. Brown.

- 3097/1. Rhombolytrum Link.

- 3119 add. Galeottia Rupr.

- 3121 vid. 2919d.

(B. Cyperacea e.)

n. 3122 add. Phyllostachys Torr.

- 3127 - AulacorrhynchusWst.

- 3135 - Choriozandra Std.

- 3126 - Crisistrix Bosc.

- 3138 - Cryptanguina Lindl.

- 3138d leg. Trachy lo m a Nees.

- 3138f add. Osmoscleria Lindl.

- 3142/1. Ptychocarya R. Brown.

- 3149 leg. Becquer elia Brgn. Becquerela Nees.

- $3151 \mathrm{~g}$ add. Diplochaeta Wst.

- 3160 - Asterochoete A. Rich.

- 3173 - Bueckia A. Rich.

- 3174 leg. Lepi d o to s perma R. \& Seh.

- 3174/1 ? Trichoballia Presl.

- 3179 add. Astroschoenus Lindl.

- 3180 - Vicentia Itr.

- 3182 - Trachyrhyngium Std.

- 3185 - Anthrostylis Dtr.

- 3189 - Isochoenus Lindl.

- 3194 - Lampocarpya Spreng.

- 3200 - Hypelythruin Dtr.

- 3215 - Vautheria Wst.

- 3216 - Ficinea Wst.

- 3219 - Fimbrystylis Dtr.

- 3229 - Heleophylax Std.

Heliophylax Ej.

? Clavula Dumort. 
Enantioblastae, Helobiae, Coronariae, Artorhizae, Ensatae.

n. 3229 b add. Baeothryum Std.

- 3230 - Mitrocarpa Torr. Somphocarya Ej.

- $3230 \mathrm{~b}$ - Heleogonus IVst.

- 3233 - Dulcichium Wst.

XIII. Enantioblastae.

n. 3250 add. Alephia Dumort.

- 3273 - Lygnia Dumort.

- 3280 - Eligia Dumort.

- 3282 - Calostrophus Wst.

- 3288 - Paleoxyris Brgn.

- 3289A .. Eriocaulon Lindl.

- 3293 - Toninia Wst.

- 3294 - Philodyce Std.

- 3294/1. Stephanophyllum Guill.

- 3295 add. d. Nematopus Seub.

- 3298 - Comelina Bunge.

- 3299/1. Dictyospermum Wight.

- 3299/2. Heterocarpus Wight.

- 3308 leg. Tin a ntia Scheidw. Tiunantia Endl.

- 3318/1. Dichoespermum Wight. Dichospermum Müll.

\section{Helobiae.}

n. 3327/1. Maundia F. Müll.

- 3328 add. Aponogetoa Dumort

- 3329 - Ouviandra Dumort.

- 3330 - a. Eualisma Seub.

- 3330/1. Baldellia Parlat.

- 3336 leg. Hydrocleys Comm. Rich.

Hydrocleis Rehb.

\section{Coronariae.}

n. 3345c add. Cephaloxyes Spach.

- 3362 leg. Acoridium Nees.

- 3368 add. Schaenocaulon Benth.

- 3369 - Asagraya Wst.

- 3381 - Wurmbaea Std.

- 3385 - Kreyssigia Wst.

- 3387 - Hexorina Std.

- 3399 - Buchosia Std.

- 3400 - Pontederas Hfmsg. Unisemma I). G.

- 3402/1. Limnostachys F. Müll.

- 3410 add. Calocorthus Dumort.

- 3410b - Cyclobothria Dtr.

- 3410/1. Eucrinum Nutt.

- 3411/1. 'Tozzettia Parlat.

- $3414 a$ add. Amblirium IIst.

- 3418 leg. Funckia I) um ort.

- 3419 add. Maulia Wst.

- 3424 - Triteleja Endl.

- 3432 - Sanseveria Dumort.
Salmea Wst.

Pleomeles Salisb.

n.3433 add. Trithomanthe Wst.

- 3434a - Aprica Wst.

- $3434 \mathrm{~g}$ leg. Pachydendron D u $\mathrm{m}$.

- 3435 add. LomathophyllumDm.

- $3436^{\circ}$ - Codonocrinum Willd.

- 3442 - Weltheimia Brgn.

- 3443 - Litanthes Iindl.

- 3449 - Peribaea Lindl.

- 3451 - Drimya Lemr.

- 3457/1. Nectaroscilla Parlat.

- 3458b add. Urgenia Wight.

- 3460/1. Caruelia Parlat.

- 3464 del. Strangweja Bert.

- 3464/1. Foxia Parlat.

Strangweja Bertol.

- 3466 add. Gardinia Bert.

- 3468 - OrnithogaloideunDtr.

- 3469 - Soberbaea Dtr.

- 3474/1. Bidwillia Herb.

- 3489 add. Stipandra Dtr.

- 3496/1? Chatocapnia Sweet,Std.

- 3496/2 ? Gyrenia Knowl. \& W.

- 3502 add. 'Tulbachia Itr.

Tulboghia Wst.

- 3505 - Rhyacophila Wst.

- 3524 - Xanthorhaea Dumort.

- 3538 - Irillium Rafin.

- 3545 - Majanthemum Siegesb.

- 3546/1 ? Brachypetalum Nutt., Lindl.

- 3562/1. MajanthemophyllumVis. \& Mass.

- 3563 add. Phylesia Dumort.

- 3574 - Turpistra Std.

\section{Artcrhizae.}

n.3581 leg. Jan-Raia Plum. Janraja Adans.

- 3582 add. Oncorrhiza Std.

\section{Ensatae.}

n.3587 add. Diplandra Bert.

- 360:3/1 ? Luchia Std.

- 3604 ardd. a. Euburmannia Seub.

b. Tripterella Rich.

- 3605 - Gonianthes Dtr.

- 3612 - Gymnosyphon Dtr.

- 3617 - Gálathea Salisb.

Orthosanthus Std.

- 3618 leg. Cruilishankia Rchb.

- 3618/1. Symphyostemon Mrs.

- 3618/2. Psythirisma Herb.

- 3618/3. Echthronema Herb. 
n.3618/4. Eriphilema Herb.

- 3618/5. Calydorea Herb.

- 3618/6. Glumosia Herb.

- 3619 add.Sisyrinchium R.Brown.

- 3620 - Hydrastylis Std.

- 3621/1. Plantia Herb.

- 3621/2. Trimezia Herb.

- 3623 add. Helixira Std.

- 3626 - ? Sisynrichiuin Herm., Burm.

Juno Tratt.

- 3626e - Xyridium Std.

- 3628 - Tinantia Dumort.

- 3632 - Tigrida l)umort.

- 3636 - Aristaea A. Pich.

- 3638 - Pattersonia Wst.

- 3640 leg. Merist ostigma Dtr. Meristastigma Std.

- 3641 add. Anomotheca Leinr.

- 3644 -. Sphaerospora Sweet.

- 3646 - Watzonia Iumort.

- 3649 - Freesea Std.

- 3653 - Geissorrhiza 1)tr.

- 3654 - Nemastylis Lindl.

- 3656/3 ? Lomenia Pourr.. Vitr.

- 3658 add. Hachenbachia Dtr.

- 3659 leg. Xiphidium Löfl.. Aubl. Xyphidium Neck.

- 3665 add. Heriteria Dumort.? Camderia Ej.? Pyrotheca Std.

- 3076 - b. Euvellosia Seub. Telosia St. Hil. Raddia Lindl.

- 3681 - Galactanthus Lemr.

- 3683 - Sceptranthes Std.

- 3687 - Habrantus Dumort.

- 3694 - Eustrephia 1)tr.

- 3697 - Valota Dumort.

- 3701 - Lycorus Loud., Std.

- 3704 - Hinanthophyllum Dtr.

- 3707 - Boophana Std. Bouphone Lemr.

- 3713 - Carpoliza Std.

- 3717 - Liperiza Lindl.

- 3721 Leperrhiza Wst.

- 3721 - Callithama Wst.

- 3744 - Alstromeria Lindl.

- 3748 - Chaeradodia Lindl.

- 3748/1. Agallostachys Beer.

- 3751b add. Littlea Dumort.

- 3754 leg. Polyanthes Ej. Pothos Adans. (Std.)

- 3755 add. Robynsia Drap. Zetocapnia Link \& 0 .
Caetocapnia Std.

n. 3756 add. Doratanthes Lemr.

- 3761 leg. Bilbergia Du mort.

- 3760/1 ? Lamprococcus Beer.

- 3761/1 add.Macrochordium Beer.

- 3761/2 ? Echinostachys Beer.

- 3762 add. Pothuava Gaudich.

- 3766/1 ? Pityrophyllum Beer.

- 3770 add. Vriesea Beer.

- 3771 - Brochinia Std.

- 3772 - c. Orthophytum Beer. Orthopetalum Ej. d.Lamproconus Lemr.

- 3773 vid. 3772 d. Phlomostac hys Beer.

- 3775 - 3772d.

- 3777 leg. Rena lmia Plum. Renealmia Linn.1737.

- 3777/1. Phytarrhza Vis.

- 3786 add. Pouretia Lindl.

- 3788 leg. Platystachys C. Koch. Allardtia litr.

- 3759 vid. $3772 \mathrm{c}$.

- 3793/1 add. Chevaliera Beer.

- 3793/2 leg. Quesnelia Gaudich. Guesmelia Auct.

- 3793/4 del. $=3770$.

\section{Gynandrae.}

n. 3803 add. Asperegrenia Spach.

- 3815 - Eveleyna Std.

- 3820 - Nephelephyllum Ej.

- 3821 - Corallorrhiza Gmel.

- 3823/1 ? Distichis Pet.-Th.

- 3834/1 ? Dichopus Blume.

- 3835/1. Oxysepaia Wight.

- 3838 add. 'Tribachia l'umort.

- 3843 leg. Xiphizusa Rchb. f.

- 3845/1. Theiy chiton Endl.

Thelichiton Duch.

- 3847 add. Nicrocaelia Ej.

- 3851 - Phreatea Wight.

- 3852 - Lichinora Wight.

- 3852/1. Conchidium Gritt.

- 3852/2. Aggeianthus Wight.

- 3855 add. Schismocerus Std.

- 3858 - Metachilon Std.

- 3861 - Hexopea Std.

- 3865/1 ? Pattonia Wight.

- 3869 add. Anacheilum Wst.

- 3875 - Diothonaea Leinr.

- 3875/1. Gastropodium Lindl.

- 3878 add. Leptothrium Kth.,Std. Eleanthus Wst.

- 3880 leg. Nemiaconia Know 1.

- 3883 add. Cattleia Std. 
n.3884 leg. Barkeria K n ow l.

- 3885 add. Cyanorchis Pet.-Th.

- 3888 - Spatoglottis Std.

- 3889 - Thiebaudia Wst.

- 3894 - Arundenia Wight.

- 3895 - Phaios Wst. Phajas Wight.

- 3903 - Plocaglottis Std.

- 3905 - Physingia Stud.

- 3908/1. Dignathe Lindl.

- 3915 add. Orchidofunkia Iindl.

- 3927/1. Promenaea Lindl.

- 3934/1. Scuticaria Lindl.

- 3936/1. Stenocoryne Lindl.

- 3939 leg.Tetragamestus R c h b.f.

- 3946b add. Monacanthus Sweet.

- 3947/2. (lowesia Lindl. Clovesia Wst.

- 3952 add. Corythanthes Lemr.

- 3959 - Trichorrhiza Lindl.

- 3968 - Broomheadia Wight.

- $\$ 969$ leg. Ansellia Lindl.

- 3972 add. Cistela Blume. Ascochilus Fj.

- 3981b vid. 3981/1.

- 3981/1. Zygosepaluin Rchb. f. Zygosepalon $\mathrm{Ej}$.

- 3997 add. Rodrigueza Dumort. Rodriguesia Brgn.

- 3998 - ? Physanthera Bert.

- 4007 - ? Coppensia Dumort.

- 4008 leg. Leochilus Knowl.

- 4018 - Macrochilus K nowl.

- 4025/1. Cottonia Wight.

- 4040 add. [a. Selenostylis Blume. b. Scalpellorchis Fj.]

- 4044 add. Aeceociades Duch. Aeceolades Wight.

- 4055b - Ghiesbrechtia Lindl.

- 4061 - Euphrobosces Wight. Euproboscis Richb. f.

- 4061/1. Josephia Wight.

- 4071 leg. Schlimia Planch.

- 4076/1. ? Epithecia Knowl. \& W.

- 4077 add. Dactylorrhiza St.d.

- 4093 - Peristylis Wight.

- 4113 - Dissorrhynchium W'st.

- 4106 leg. Diplo ch il os Lindl. Diplochilus Std.

- 4112 add. Bartholinia Dumort.

$-4126 \mathrm{~b}$ leg. Isias Not.

- 4132 add. Diseris Wight. Diperis Iij.

- 4133/1. ? Nitostigma Blume.

- 4134 add. Gastrodium Dumort. n.4140 add. Schönleinia Klotzsch.

- 4154 leg. R o p trostemon Blume. Rophostemon Endl. Rhophostemon Wst.

- 4155 - Prescottia Lindl.

- 4156/1. Govindooia Wight.

- 4163a add. Aristotelia Wst.

- 4165 - Stenorynchus Rich.

- 4168 add. CheiradoplectrumWst.

- 4169 - Plexaura Wst.

- 4171 - Zeuxina Std. Zuxine Wight.

- 4179 leg. Neottia Rebent.

- 4188 add. Ucantha Duch.

- 4192 - Psychechilus Wst.

- 4199 - Genosplesium Duch.

- 4206 - Cyrstostylis Dumort.

- 4209 - Eriochylus Std.

- 4212b - Elytranthe Lindl.

- 4213 leg. P t e r o stylis R. Brown.

- 4214 add Liperanthus Duniort.

- 4217 leg. Calaena Lindl.

- 4221/2. Didymoplexis Griff.

- 4226 add. Gavillea Std. Gavila Wst.

- 4227 - Bipennula Std.

- 4229 - Lidmodorum Dumort.

- 42:39/1. Apetalon Wight.

- 42:39/2. Podanthera Wight.

- 4241) add. Arietinium Beck.

- 4245 - Calista Ritg.

- 4245/1 vid. 3947/2.

- 4249/2. Physarus Lindl.

- 4250 add. Pleuroblepharon Std.

- 4251 vid. $3845 / 1$.

- 4251/4. Scaredederis Pet.-Th.

- 4251/5. Eicosia Blume.

- 4255 add. Rhyncanthera A. Rich.

XIX. Scitamineae.

n.4256d add. Sphaerocarpus Juss.

- 4257 - Dietrichia Räusch.

- 4259 - Aro-Orchis Burm.

- 4259b - Trilophus Lindl.

- 4261 - Ettlingera Std. Hornstedia Juss.

- 4263 - Dirhacodes Lemr.

- 4265 leg. A I pina Plum. Alpinia Gärtn.

- 4274 add. Monocistis Std.

- 4279 - b. Saranthe Reg. \& K.

- 4286 - Canacorus (Endl.) Wst.

\section{Fluviales.}

1.4300 add. Nagas Gis.

- 4306 - Podisonia Dumort. 
10 Spadiciflorae, Principes, Coniferae, Piperitae, Aquaticae, Juliflorae.

n.4311/1. Grönlandia Gay.

- 4311/2. Spirillus Gay.

- 4314 vid. 3587.

- 4315/1. Schizothec'a Ehrenb.

- 4320 add. Bibliolithes Schloth. Phyllites Brgn.

- 4322 - Hydrophaca Hall.

\section{Spadiciflorae.}

n.4325 leg. Kodda-Pail $\mathrm{Pl} \mathrm{u} \mathrm{m.}$

- 4329 add. Cryptocorina A. Rich.

- 4332 - Criptocoryne

- 4333 - Ariscema Std.

- 4347 - Thompsonia Std.

- 4354 - Colocassia Wight.

- 4359 - Xanthosmia Std.

- 4365 - Philodendrum Std.

- 4388 - Stathiphylluin A.Rich.

- 4391 - Symptocarpus A. Rich.

- 4399 - Sparangium Ritg.

- 4407/2. ? Pandanophyllum Walp.

- 4408 add. Carludowica Wst.

- 4416/1. Podocarya Buckl.

- 4418/1. Vinsonia Gaudich.

\section{Principes.}

n.4424 add. Kuntia Durnort.

- 4438 - Drypsis Duch.

- 4439 - Seafforthia Std.

Psychosperma Dumort.

- 4441 - Lirymopalaeus Lindl.

- 4442 leg. Saguaster minor Rumph.

Seguaster minor Auct.

- 4449 - Saguaster major Rumph.

Seguaster major Auct.

- 4454 add. Daemonorophus Lemr.

- 4456/1. Cálamosagus Griff.

- 4476/1. ? Cryosophila Blume.

- 4478 leg. Cham a eriphes Pont., Gärtn.

- 4481 add. Trinax Dtr.

- 4482 - Fulchironia Lestib. Phaenix Dumort.

- 4491 - Lithocarpos T'arg.

- 4494 - Coccus Linn. Palma Plum.

- 4508 leg. Endogenites Br on g n.

\section{Coniferae.}

n.4519 add. Platyclados W'st.

- 4524 leg. R e t in ispora Sieb. \&Z.

- 4540 - Taxodites Presl.

- 4540/1. Glyptostrobites Brgn. n.4540/2. Cryptomerites Brgn.

- 4540/3. ? Eleoxylon Brgn.

- 4545/1 add. Melitoxylon Hart.

- 4546a - Tsuja Zucc.

- 4547a - Columbea Std.

- 45.53 - ? Saxe-Gothaea Lindl.

- 4554A - Abies Brgn.

- 4554B - Pinus Brgn.

- 4561 leg. Dammarites Presl., Sternb.

Dannarites Findl.

- 4568/1. Sequoites Brgn.

- 4589 vid. $4545 / 1$.

\section{Piperitae.}

n.4599 add. Pepronia Dumort.

- 4612e - Leiophyllum Wist.

- 4617 leg. Saururus Pluin.

\section{Aquaticae.}

n.4617 add. Tulasnea Wight. T)alzellia $\mathrm{Ej}$.

\section{Juliflorae.}

n.4653 add. Chamelaeagnus Dod.

- 4653c - Asplenium Stermb.

- 46lia $\beta$ leg. Cerroides Spach.

- 4675 leg. Pliegonium Ung. Fegonium Ej.

- 4680 add. Holoptelaea Ej.

- 4686d - Colletia Scop. e? Momisiopsis Blume

- 4687/1. Homoioceltis Blunie.

- 4688 add. Trema Lour.

- 469i/1. Galumpita Blume.

- 4694 add. Morophorus Std.

$$
\text { Ampalus Wst. }
$$

- 4696 - Machura Std.
a. Eumaclura Blume.

b. Leptosura Ej.

- 4697. Bleekrodea Blume.

- 4708 add. ? Coilotapalus P. Browne.

- 4711 - Corocephalus Ditr.

- 4718/1. ('ephalotrophis Blume.

- 4719 add Bucephalum Std.

b. Parastreblus Blume.

- 4719/1. Paratrophis Blume.

- 4719/2. Taxotrophis Blume.

- 4724 add. Peribea Dumort.

- 4731 - Anthiaris Dumort.

- 4744 - a. Urticella Miq.

474 b. Chamaecnide Ej.

- 4747 - Elatostoma Wight.

Langefeldia Std.

- 4752/1. ? Leptocnide Blume. 
n.4763 add. Urtica Burın.

Bohmeria Dumort.

e? 'Tilocnide Blume.

f ? Urocnide Ej.

g? Sarcocmile $\mathrm{Ej}$.

- 4764/1. Stachyocnide Blume.

- 4767 add. Gesnouisia Std.

- 4775 leg. Forskohlea Linn.

Forskolea S chreb.

Forskoelea Brgn.

Forskolia Wight.

Caidbeja Forsk.

Caidbeia Std.

- 4776 add. Drogouetia Std.

- 4780. - Moldenhaueria Std.

- 4781 - Theligonis Reuss.

- 4785 - Fadgenia Lindl.

- 4796 leg. Henslovia A. Juss.

- 4798 add. Naemotospermum Std.

\section{Oleraceae.}

n.4\$06 add. Roubieua Carey. Roubiaeva Brgn.

- 4807 leg. b. Morocarpos Rupp.

- 4810 - Bosia E.j.

add. Bosaea Duniort.

- 4816 - Theleophytun Wst.

- 4827 - Kirilovia Lindl.

- 4828 leg. Camforosma M e yer. 4832 add. ('helona Dumort.

- 48.85 - Willemeta Gmel.

- 4846 - Fremontea Lindl.

- 4851 leg. Schoberia M eyer.

- 4864 add. Halogeiton Wst.

- 48i2 - Cuscuta Linn. Cliff.

- 4877 - Beriesa Std.

- 4878 - C'ladostachis std.

- 4981 - ? Amaranthoides Turm.

- 4883 - Kokera Adins. Chammisoa Dis.

- 488ib leg. Pyxidium Moy.

- 4897 add. Ptilothus Dumort.

- $4 \times 99$ - Gomotriche Kj.

- 4901 - Ourèt Adans.

- 4901/1. Pseudanthus Wight.

- 4904 leg. Achiranthes P. Browne. add. Achrantes Dumort.

- 4919a - Tromsdorffia Std.

- 4921 leg. Gomphraena J a c q.

- 4921 a add. Serturnera Auct.

- 4922 - Fröhlichia Endl.

- 4931 - Mucrenia Std.

- 4943 - Centopodium Std.

- 4944c - Rumastra Wst.

- 4945 - Atraphraxis Brgn.

- 4945c - Tragopyron Wst. n.4949d add. Amblyogonum Wst.

- 4951 - Frumentum Matth.

- 4954 leg. Coccol o b is P. Browne. add. Schlosseria Mill

Conobaea Bertero.

- 4958 - Vellaquezia Wst.

- 4960 - Antigonium A. Juss.

- 4968 - Oxybaphos Wst.

- 4975 leg.B o u gainvillaea Choisy. Bugainvillaea Brgn.

- 4979 add. Torrulia Std.

- 4982 - Plumbaginoides Boerh.

\section{Thymelaeae.}

n.4989 add. Citrosena Bosc.

- 4991 -' 'Tetrapome Duch.

- 4997 - Antherosperma Poir.

- 4999 - Doratophora Lemr.

- 5015 - Eudiandra Dumort.

- 5019c - Gomortegia Wst.

- 5024 - Evonymadaphne Wst.

- 5026 - Misantheca A. Juss.

- 5027b - ?Perostema Räusch.

- 5030 - Pleorothyrium Endl.

- 5036 - Camphoromaea Leinr. Camphoromea A.Juss.

- 5052 leg. Litsaea Nees.

- 5063 - Scleropyrum Wight. add. Halmiltonia Dumort. Calinux Std.

- 5069 - Linophyllum Pont.

- 5071 - Balenerdia Std.

- 5075/1. Eucarya Mitch.

- 5089 leg. Schoenobiblus Mart. Schoenobiblos End!.

- 5099 add. Days Dumort.

- 5100 - Edgworthia Lindl.

- 5101 - Enkleia Gr., Lindl.

- 5119 - Hernandezia Hfmsg. Hertella std.

- 5123 - Gyrinopsis Lindl.

- 5125 - Xyloaloë Rumph.

- 5129 - Lepargyraea Std.

- 5131 - Elaeachnus Burm.

Elaeagrus Pall.

- 5139 - Geisoloma Ej.

Gassoloma litr.

- 5143 - Leucandron Std. Erodendron Wst.

- 5144 - Deastella Kn., Std.

- 5150a - Mischocaryum Wst.

- 5159 - Cennarrhenes Std.

- 5160 - Potamica Poir.

- 5161 - ?c.Sacculigera Lindl.

- 5163 - Brabeum Beckm, 
n.5164 add. Andriopetalum Lemr. Panopis Std.

- 5169a - Plagiopodia Dtr.

- 5174b - Amphiderrhis Lemr.

- 5175 leg. Rupala Vahl.

- 5176 add. Adenostephanes Lindl.

- 5177 - Helitophyllum Wst.

- 5179 - Catas Juss., Std.

- 5180 - Catas Juss. pt.

- 5185 - Driandra I)umort.

\section{Serpentariae.}

n.5197 add. Trimerizia Std.

\section{Aggregatae.}

n.5217 add. Nardus Garc.

- 5220 - ? Oligacoce Willd.

- 5229 - Diolotheca Rafin.

- 5234b - Astrocephalus Dumort.

- 5236/1. Nastanthus Miers.

- 5239 add. Echinoloma Std. Somea Wst.

- 5252b - Protolepis Std.

- 5252f - Oliganthus Wst.

- 5252h - Cacalia Burm.

- 5252i - Scabiosa Burm.

- 5286e - Pseudoelephantopus Std.

- 5293 - Noccea Dumort.

- 5297 - Bojera Std.

- 5311 - Lorentia Sweet.

- 5312/1. ? Pontesia Arrab.

- 5314 arld. Eupiqueria Gardn.

- 5328 leg. Stevia C'avan. Mustelia Spreng.

- 5339 add. Disynapheia Std.

- 5343 - Decachaita Wst.

- 5349b - Brachycalymna Wst.

- 5354 - Bustamenta Alam.

- 5:378b leg. Polyarrhena Ciass.

- 5381 add. Bellidaster 1)umort.

- 5382 - Virgaria Rafin.

- 5390 - Callimeris Spach.

- 5409 - Callestephus Wight.

- 5415 - Melanodendrum Std.

- 5417 - Vittadina Walp. Vittadenia Std.

- 5422b - Terrana Wst.

- 5435 - Chaetopappus Sch. Bip.

- 5446 - Hunefeldia Lindl.

- 5459 - Garale um Walp.

- 5469 - Amoleiachyris Walp.

- 5477 - Bradburya Wst.

- 5479 - Woodwillea Walp.

- 5480 - Psiodia Walp.

- 5492 - Amphirhaphis Walp.
Amphirapis Lindl.

Amphirrhapis Wst.

1.5496 add. Comuidendron Lemr.

- 5500 - Hoorebeckia Std.

- 55012 - Macrocnema Lindl.

- 5503/1 ? Cyclopappus Cass.

- 5505 add. Lisosyris Brgn.

- 5508a leg. Scepinia N e ck.

- 5511 add. Duhaldia Sch. Bip.

- 5528 - Gymnarhena DC.

- 5533 - Laenecia Walp.

- 5534a leg. Andryaloides DC.

- 5534b - 1)imorphanthes Cass.

- 553f add. Phagnalion Wst.

- 5539 - Elachothamnus Wst.

- 5542 - Pterocladis Lamb.

- 5542/1.Achyrobaccharis Sch.Bip.

- 5546/1 ? Döllia Sch. Bip.

- 5549 add.? Sphaeranthoides Cun.

- 5556 - Figalonopsis Wst.

- 5560 - Calymnandra Lindl.

- 5561 leg. Epaltes Cas s.

- 5563 add. Denneckia Std.

Deneckia Sch. Bip.

- 5566 - Rhantherium Sch.Bip.

- 5568e - Erithia Wst.

- 5572 - Karthemia Walp.

- 5573 - Jacobaea Burm.

- 5580 - Pieraea Walp.

- 5581b leg. De ca chaeta Jaub. $\& \mathrm{Sp}$.

- 5591 add. Caesulea Wight.

- 5593 - Ulek Walp.

- 5594 - Astericus Lindl.

- 5595 - Dontospermum Sch. Bip.

- 5597 - Cervillea Sch. Bip.

- 5600 - Cryptodia Walp.

- 5605 - ? Caltha Burm.

- 5606 - Blainvillia Wst.

- 5610 leg. Siegesbekia All.

add. Sigisbeckia Dumort.

- 5611 - Wiborgia HBK. Viborgia Std. Baziasa Std.

- 5629 - Rincourtia Std.

- 5632 - Ichtyothere DC. Ichthyothera Wst.

- 5633b - Thrixis Wst.

- 5633c - Thrixidium Wst.

- 5335 - Blennospermum Decne.

- 5642 - Tylocarpus Wst.

- $5646^{\circ}$ - Espletia Std.

- 5653 leg. Cer atogyne Turcz.

- 5655 add. Pinellosia Lindl.

- 5668c - Argyrochaita Wst. 
n.5671b add. Skirrhoglyphis Wst.

- 5674 - Phyllactis Std

- 5675a leg. Crassina Sc ep.

- 5682 add. Eleuthrantheron Strl.

- 5688b - Helenonoium Willd. Helemonium Std.

- 5692 - Werinnua Heyn.

- 5698 - Zalucania Std.

- 5703 - Dracopsis Lemr. Dracontopsis Ej.

- 5704 - Lepachis Std.

- 5708 - Gymnoloma Bartl.

- 5709 - Gymnolomia Wst.

- 5711 - Scleropappus Brgn.

- 5712 - Enchelya Lemr. ? Eucalia Räusch.

- 5716 leg. Diplosás t er a Tausch. Diplosastra Endl., Wst.

- 5719 add. Epilepsis Lindl.

- 5721 - Leachea Std.

- 5725c - Pterophytum Wst.

- 5739 - Dolichotheca Ej.

- 5740 - ? Agrimonia Rumph.

- 5743 - Adenocarpus Wst.

- 5746 - Perimenium Std.

- 5753 leg. Hingstonia Ej. Hinghstonia Std.

- 5753a - Phaethusa Gärtn. Phaetusa Schreb. Cle rodendron Ht. it.

- 5775 add. Gymnactis Ej.

- 5781 - Navenburgia Juss.

Nauemburgia Std.

- 5787 - Lebetinia Std.

- 5788c - Boeberoides Std.

- 5791 - Belenidium Arn.

- 5807 - Leoptoda Dumort.

- 5809 - Guiterrezia Walp. Guttierrezia Wst.

- 5821b - Macrocephalus Lindl.

- 5825 - Cercostylus Wst.

- 5827a leg. Euhymenoxy s DC. Euhymenoxis Endl.

- 5837 ardd. Picardenia Std.

- 58:38b - Mesotetra Wst.

- 5839 - Amblylepis Decne.

- 5845 - Argyrophytum Wst.

- 5851b - Chrysosphaerium Willd.

- 5851c leg. Caleacte R. B rown.

- 5853 add. Vasargia Std.

- 5854 - Vigolina Poir.

- 5857 - Thridax Beckm.

- 5861 - Thelythamnus Wst.

- 5891 - Chrysopelta Tausch.

- 5894a = ChamaecyparissiasWst. n.5894b add. Babounga Wst.

- 5896 - Eriocarpa Lag.

? Eriosphaera Dtr.

- 5900 - Spyridanthus Wst.

- 5903 - Conogyne Wst.

- 5938f - Rhytidocarpaea Wst.

- 5949 - Peurousea Std.

- 5951 - Ananthocyclus Wst.

- 5956 - Homalanthus Wst.

- 5960a - Microrrhachis Wst.

- 5960c - Cenorrhachis Wst.

- 5962 - Bembecodium Lindl. Bembicodium Wst.

- 5964 - Morusia Brgn.

- 5977a - Asteringa E. Meyer.

- 5987 - Plagiochilus Lindl. Plagiocheilos Wst.

- 5998 - Hirnelia Wst.

- 6038 - Rhytidosis Wst.

- 6044 - Leptorrhynchus Wst.

- 6052 - Scalpiopsis Std.

- 6059 - Eriosphaeria Wst.

- 6064f - Sericophysum Std.

- 6068 - Freemannia Std.

- 6073 - ? Dasyranthus Raf.

- 6086 - Pachyrynchus Std. Pachyrrhynchus Wst.

- 6088b - Leiachnaea Std.

- 6093a - Selago Burn.

- 6099 - Argyranthus Wst.

- 6100a - ? Dysinanthus Raf.

- 6100b - Ptilachis Raf.

- 6104b - Pseudoathrixia Wst.

- 6105 - Antitrixia Decne.

- 6106b - Longchampsia Wst.

- 6109a - Montagnia Spreng.

- 6110 - Amblycarpum Lemr.

- 6126a - Ptileris Raf. Neocies Std.

- 6126b leg. Psiloderis DC. Microderis Ej.

- 6130 add. Emelia Wight.

- 6145 - Synosma Raf.

- 6150a - Cacaliastrum Std.

- 6156 - Brachyrrhynchus Wst.

- 6156a - Eubrachyrhynchus Std. Eubrachyrrhynchus Wst.

- 6166 - Crassula Mill.

- 6169 - Calendria Reuss.

- 6183b - Lycotis Hfmsg.

- 6185 - Cleithria Std.

- 6188 - Aloiozonium Lindl.

- 6189 - Laudtia Wst.

- 6198a - Christea Wst.

- 6202 leg. Apule ja Gärtn. Apuleia Less. 
n.6203 add. Echinopsis Linn.

- 6210 - Bennetia Std.

- 6i210a del. Cyathidium ('ass.

- 6210b ard. Benedicta Wst.

- 6212b - Hemistephia Std.

- 6212c - Eriocoryna Std.

- 6224 - Ancanthia DC.?. Buek.

- 6229d - Pararhysis Endl. Pararrhysis Wst.

- 6229e - Amblypogon Decne.

- 6233c - Anaphalanthus Wst.

- 6236h - Leptranthus Std.

- 6236u - Veltis Adans.

- 6236y - Mesocentrum Std.

- 6236aa - Leucacantha Wst.

- 6236cc leg. Seridia DC.

- 6242b add. Odontagnanthia Std.

- 6243 - Cartamus Beckm.

- 6246 - Sylibum I)umort.

- 6248 - Tyrimmus Bartl.

- 6249 - Onopyxus Dalech.

- 6254 - d? Chromolepis Cass. del. Veltis Adans.

- 6256 add. Pycnocomon Wst.

- 6257 - Crisium Lachen.

- 6258 - Epitrachis Wst.

- 6258b - Phoenix Lobel.

- 6260a - Ptilostemum Wst.

- 6250b - Polyacanthus Bauh.

- 6271 - Jurinaea Std.

- 6275 - Bardanesia Durnort.

- 6285 - Centroclinion Orb.

- 6288 - Gochnatea Std.

- 6288a - Cyclopsis Wst.

- 6288a $\beta$ - Anastrephia Decne.

- 6301 - Brachycladus Wst.

- 6302 - Chaenanthera Dumort.

- 6333 - Chevreuilia Lindl.

- 6336 - Leptalea Don.

- 6343b - Piptostemum Std.

- 6343c leg. Eup a n a r g yruin DC.

- 6349 add. Penthanthus Std.

- 6359 - Castra Arrab.

- 6359a - Holocheilos Wst

- 6359b - Oligophyllum Wst.

- 6359bi - Polyphyllum Wst.

- 6361 - Dolicholasium Lemr.

- 6371 - Ragadiolus Beckm.

- 6382 - Henselera Rchb. Hänslera Std.

- 6383 - Intybus C. Bauh. Endivia $\mathrm{Fj}$

- 6394 - Amblachenium Wst.

- 6405 - Asterotrix Brgn. Asterothria Gren. n.6409 add. Gerontopogon Gesn.

- 6411 - ? Richardia Roth.

- 6432 - ? Jamesia Necs.

- 6436 - Brachyrrhamphus Wst.

- 6445 - Zacynthia Peckm.

- 6446a - Catyona Lindl.

- 6449 - Microrhyncus Wight. Microrrhynchus Wst.

- 6450 - Macrorrhyurus Brgn.

- 6455 - Barckhausenia Menke. Barkausia Brgn.

- 6458d - Psilochaena Walp.

- 6466 - Heteracea Std.

- 6468 - Andriala Decne.

- 6475a - Micrauchenia DC.

- 6481 - Troxinum Std.

- 6501/1. ? Cynura C'ass.

- 6503 add. Dimenostemma Std.

- 6513 - Mumrozia Std.

- 6520/1. Dadia Arrab.

- 6520/2. Epilatoria Comm.

- 6524/1. Orcya Arrab.

- 6525 add. ? Osteiza Llav., Std.

- 6525/1. Sabbata Arrab.

- 6525/2 Torrentia Arrab.

- 6526 add. Teichostemma Std.

- 6526/1. Xenophontia Arrab.

- 6527 add. Xantheum Wight.

\section{Campanulinae.}

n.6541 add. Boutonia H. Erf.

- 6547 - Velleja Endl.

- 6550 - Calligyne Lemr.

- D561 - Drobowskia Brgn.

- 6565a $\beta$ - Dortmannia Std.

- 6568 - Siphocampylos Wst.

- 6572 - Isostoma Dtr.

- 6590 - Campanuloides H. Kew. Ligfootia Dumort.

- 6601 - ? Roëlloides Banks.

- 6t004 - Phiteuna All.

- 6614 - Muschia Brgn.

- 61522 - Leeurenhockia Std.

\section{Caprifolia.}

n. 6627 add. Vallantia Dtr.

- 6630a - Staurogalium Std.

- 6637 leg. Scherardia Murr. add. Sherhardia Gled.

- 6641 - Phillis Allion.

- 6642 leg. Caprosma Dumort.

- 6646 add. Scyphyphora Dtr.

- 6651 - Bucholzia Wst.

- 6655 - Spermacocce Dumort.

- 6676 . Cruckshankia Std. 
n.6677 add. Acrochordodendros Pluk.

- 6680 - Uuragoga Linn.

- 6684 - Sutoria A. Juss.

- 6692 - Dychotria Rafin.

- 6696 - Mapourea Wst.

- 6699 - Carpothales Liıdl.

- 6700/1. Codonocalyx Miers.

- 6704a leg. 'Tar amea Rafin.

- 6704/1. Macrocalyx Miers.

- $6700^{\circ}$ add. Grumelia Wight.

- 6707 - Polyoxus A. Juss.

- 6711 - Jasininum Burm.

- 6718 - Chiocca Dtr.

- 6726 - ? Lycioserissa Riöm. \& Sch.

- 6729c - Psydax Std.

- 6732 - Eurynome DC. Eurynioma Std.

- 6733 - Aniarocarpus Wst.

- 6735 - Poederia Reuss.

. 6737 - ? Disodia Dumort.

- 6746 - Geoherpum Willd.

- 6753b leg. Langeria Linn.

- 6774 - Ancylan thos Desfont. Ancylanthus Juss. Anclyanthus A. Juss.

- 6778 - Cuvieria A. Juss.

- 6789 - Erosmia A. Juss.

- 6795 - Lachnosyphonium Lindl.

- 6803/1 Garapatica Karst.

- 6004 add. Isartia D)umort.

- 6815 - Bruinsmannia Lindl.

- 6807 - Caryococca Willd.

- 6811 - Conyza Burm.

- 6813d - Euhediotis Std.

- 6814 - Scieromitrum Wst.

- 6815 - Antirrhinum Burm.

- 6825 - Karamyschovia Std.

- 6844 - Rondoletia I)umort.

- 6850 - Hinsia Wst.

- 6855 - Bonitacia Manso.

- 6861/1. Tresanthera Karst.

- 6865, add. Pinckeneya Dumort.

- 6866 - Christima Std.

- 6873/1. ? Acrostoma Ilidr.

- 6874 add. Remigia Wst.

- 6876/1. Joosia Karst.

- 6886 add. Ferreiria Wst.

- 6889 - Courtarea Dtr. ? Outarda Dumort.

- 6897 - Crossopterix Wst.

- 6898e - Ourouparea Wst.

- 6911 leg. Stylocorina Gärtn.f.

- 6914 - Mussaenda Burm. n.6915 add. Adenosachma A. Juss.

- 6920d - Talangninia Chap.

- 6927 - Higgensia Std.

- 1;939 - Menesteria Dtr.

- 6944c - Herghias Std. Chaquepiria Ej.

- $6950^{\circ}$ - Melanopsidium Colla.

- 69 ii3 - Scepsothamius Wst.

- 6967 - Eteriscus Std.

- 6978 - Myrioneurum Std. Bertiera Hanilt. Berliera Std.

- 6979 - Pleothea Std.

- 6985 - Leicesteria Wst.

- 6990 - Triostemum Dtr.

- 6996 leg. Alsenosinia Endl.

\section{Contortae.}

n.7000 add. Nictanthes All.

- 7004biz - Bumeloides Std.

- 7007 add. ? Rangium Juss.

- 7009 - Lilacum Ren.

- 7015 - Myospyrum Lindl.

- 7027 leg. Actegeton Blume.

- 7031 add. Curare Humb.

- 7034 - Pegamea Vitm.

Pegamaea Dumort.

- 7040 - Anthelmenthica P.Br.

- 7042 - Mitrasachme Wight.

- 7045 - Anaster I)umort.

- 7047b - Picrophleus i)umort.

- 7051 - Anthoclista DC.

- 7057 - Landolfia Lind!.

- 7059 - Willugbeia Dumort.

- 7074 - Leucanotis Drr.

- 7076 - Toxicophloea Lindl.

- 7082 - Ligustrunı Burm.

- 7091 - Rauvolfia Plum.

Rauwolfia Lin n.

- 7150 - Caudicia Hamilt.

- 7152 - Haenadiction Std.

- 7155 - Rynchospermum

Lindl.

Trachelospermum

I.emr.

- 7197 - Guroa Hamilt.

? Gurua Ej.

- 7198 - Hemidemus Dumort.

- 7204 - Paphioacme Wst.

- 7212 - Pentoretia Lindl.

- 7216 - Scamone Dumort.

- 7224 - Steinheillia Lindl.

- 7230 - Menastelma Lindl.

- 7231 - Rhapistemma Std.

- 72:35 - Cordylogne Lindl.

- 7236 - Xymalobium Std. 
n.7236/1. Odontanthera Wight.

- 7247 leg. Col ostephanus Harv.

- 7257 add. Exytelma Dumort.

- 7259 - Hockea Lindl.

- 7264 - Asclepias Rebent.

- 7266 - Pentarhinum Decne.

- 7281 - Diplolepium Dumort.

- 7287 - Gonobolus Dtr.

- 7291 - Fischera Wst.

- 7307 - Marodenia Dumort.

- 7313 - Gymnima Wight.

- 7316 - Sarcobolus Dtr.

- 7335 - Riochreuxia Lindl.

- 7338 - BrachistelmaDumort.

- 7340b - Hutschinia Dtr.

- 7344 - Priaranthus Dumort.

- 7346b - Gerostemum Std. Gorostemon Ej. Gonostemum Wst.

- 7354 - Lysimachia Burm.

- 7359 - Lapethea Lindl.

- 7362 - Enstoma A. Juss.

- 7308 - Thurnhausera Std.

- 7375 - Orthostemum Std.

- 7377 - Flemingia Roxb.

- 7379 - Enicostemma Std.

- 7380 - Coutubea Std.

- 7388 - Petalostylis Lindl.

- 7:390d - Melonanthus Müll.

- 7394 - Perepusa Std.

- 7399 - Eriocoila Wst.

- 7399b - Urcophylla B.

- 73990 - Tetrorrhiza Wst.

- 7408 leg. S czuckinia Turcz. Sczukinia Wst.

- 7410 add. Halenea Wight.

- 7415 leg. Schweyckerta Gmel. Schweyckherta Ej. Schweickerta Ej.

\section{XXXY. Nuculiferae.}

n.7420 add. Lehmannia Jacq.

- 7426 - Basilium Wst.

- 7428 - Synclostemon Std. Syncollostemon Jindl.

- 7430 - Dentidea Std.

- 7431 - Colus Räusch.

- 743Th leg. Xanthioph a e Mart. Xanthiophoea Std.

- 74370 - Mesosphaerum P.Br. Mesosphaerium Std.

- 7439c add. Pseudostoechas Std.

- 7441 - Choteckia Std.

- 7442 leg. Colebrooke a Smith. Colebrookia Spreng. Colebrockia Std.
Buchanania Smith.

n.7445 c add. Elsholtia Koch.

Elschotzia Brgn.

- 7462e - Heteromelissa Duch.

- 7469d - Gymnocalyx Schmidt.

- 7476 - Ülricia Jacq.

Lepechina Dumort.

- 7481 - Leonura Ust.

- 7481c - Drymcosphaco Duch.

- 7487 leg. Zizyphora Dumort.

- 7488 add. ? Flessera Adans.

- 7496 .. Tertianaria J. Bauh.

- 7496b - Heteranthesis Wst.

- 7507 - Syderitis All.

Fracastora Adans.

- 7515a - Eriostemum Std.

- 7515c - Stachyditypus Wst.

- 7519b - GaleopdolonDumort.

- 7521 .. Yermoloffia Wst.

- 7529 - Notocheta Std.

- 7531 - Eriophytum Std.

- 7547b - Pheboanthe Wst.

- 7555 - Chlonanthes Dtr.

- 7558b - Uwarovia Lindl. Shuttelworthia Std.

- 7559a - Rhagocarpuin Wight.

- 7560 .. Stachyarpheta A.Juss.

- 7561b - Goniolippia Schau.

- 7580 - Permua Dumort.

Premma Brgn.

- 7582 - Tomex Linn. Illa ddans.

- 7586 leg. Clerodendron Burm.

- 7593 add. Ephialum Wst.

- 7594 - Holmskidia Dumort.

- 7000 - Avicenia St. Hil. Guapina Std.

- 7601 - Dissolaena lindl.

- 7605 - Campilostachys A. Juss.

- 7637 leg. Cordia Pluın., R. B rown. Pavonia Domb.

- 7637a add. Gerascanthos Std. Geraschanthus Iindl.

- 7642b - Carmorea Std.

- 7644 - Lytrostylis Wst.

- 7648c - Tournifortia Wight.

- 7651 - ? Goldenia Räusch.

- 7655 - Rotala Lindl.

- 7657c - Piptochlaena Wst.

- 7659f - Triaridium Dumort.

- 7662 - Lobostenum Wst.

- 766 fi5 - Macrotonica Std. Macrostomia Wst.

7673 leg. Symphitum Alliou. 
n.7686 add. Onosmidium Dumort. Purchia Ej.

- 768S - Molkia Dumort.

- 7689d - Gymnoreime lindl.

- 7693 - Steenhammaria Lindl.

- 7703 - Bothryospermum Std.

- 7708 - Friotrichium Lemr.

- 7732 - Marcielia Std.

\section{Tubiflorae.}

n.7751 leg. Ipomea All.

Piptostegia $\mathrm{Hoffms}$.

- 7753 - Convolvolus Beckm.

- 7754 - Aneseia Wight.

- 7757 - Calycostegia Lemr.

- 7768 - Cladostylus Wst.

- 7771 - Demidoffia Wst.

- 7787c - Eugillia Wst.

- 7792 - Cobea Dumort.

- 7792/1. Rosenbergia Örst.

- 7794 add. Diajenzia Dumort.

- 7797 - Macrocalyx Trew. Colpophyllus Ej.

- 7807 - Hydrolaea Dumort.

- 7814 - Neudorftia Std.

- 7822 leg. Acquartia R chb.

- 7828 add. Sarachira 1)umort. Jaltonia Std.

- 7829 - Calydermus Std.

- 7830c - Epetorhiza Std.

- 7839 - Treconaetes A. Juss.

- 7846 - Himenanthus Std.

- 7849 - Jabrosa Std.

- 7859 - Panzeria fimel. del.Jycioserissa Röm. \&Sch.

- 7862 add. Ianarckea Std.

- 7868 - Stramonia J. Bauh., Herm.

- 7870c - Withleya Dumort.

- 7880 - Nierenbergia Std.

- 7894 - Swenkia Hedw.

\section{Personatae.}

n.7899 add. Hateranthia Juss. Vrolickia Stil.

- 7904 - Fagelia Schwenck.

- 7909 - Alonsea I)tr. Alonzoa Brgn.

- 7917a - Cymballaria Std.

- 7917b - Kicksia Wst.

- 7918 - 'l'imbuleta Sid.

- 7926 - Pawlownia Limdl.

- 7930b - Sarodonia Std.

- 7933 - Pentestemon Dumort. Pentastemum Std.

- 7938 - Russellia Wst. n.7939 add. Freylenia Brgn.

- 7951 leg. Peliostomum kenth.

- 7953 add. Doranthera Std.

- 7968 leg. Brachyc o ry s Schrad. Brachycoris Std. Bowea Std.

- 7969a add. Heinzelıania Neck.

- 7972 - Unannea Std.

- 7974 - Hydropityum Std.

- 797i - Lafuentia Benth. Durieva std.

- 7978 leg. Herpestis Gärtn. f. Herpestes Link. Mon iera P. Browne. Monniera Pers.

Monneria Std. Hydrotrida Willd.

del. Heinzelmania Neck.

- 7981 add. Bacopia A. Juss.

- 7988 - Caeta Std.

- 7989 - Lindernia D. Don.

- 7990 - Ilogeton A. Juss.

- 7993 - Bonneya Dtr.

- 8006 - Pegostoma A. Juss.

- 8009 - Microcarpea Dtr.

- 8011 - Polypremuus Beckm.

- 8018 - Endigitalis Lenır.

- 8020 leg.Picrorhiza Royle,Benth. Picrorrhiza Wist.

- 8023 add. Calorrhabdos Wst.

- 8025i - ?Hedystachys Dumort.

- 8028 - Peripea Std.

- 8031 - Cyclium Std.

- 8033 - Hydrotiche A. Juss.

- \$038 - Leptorrhabdos Wst.

- 8039 - ? Cliytra Gärtn. f.

- 8041 - Sylvia Lindl.

- 8048 - Sopulina Juss.

- 8049 - Auleya Itr.

Anlaya A. Juss.

- 8051 - Harwaya Std.

- 8060 - Phthecrospermum

std.

Phteirospermum A. Juss.

- 8073 - Rhinantlus D. Don.

- 8080 - Sanchesia Benth.

- 8082;1. Hymenospermum Benth.

- 8037 add. 'Thumbergia Dumort.

- 8088/1. Schmidia Wight.

- 80:11 add. Elythraria 1)tr.

- 8094 - Lbermiera Wight.

- 811. - Jischoriste Dtr.

- 8115 - Calophanos Wst.

- 8120 - Neowiedia Lindl.

- 8133 - DidyplosandraWight. 
n.8142 leg. Arrhostox y lo n Mart. Arrhostoxylum Nees.

- 8158 adil Russegera Std.

- 817i - Salpixanthus Lindl.

- 8178 - Strobilorachis Lindl.

- 8178/1. Porphyrocoma Hook.

- 8202 add. J Juvernoya Wst.

- 8214 - Rytiglossa Std.

- 8222 leg. a. ('ampylostemon E. Mey.

b. Pectoraria Nees.

- 8232 add. ? Nisanthera Beckrn. ?Diptanthera Schrank.

- 8238 - Chamaeranthemum Fj.

- 8246 - Diclyptera Dtr. Diapedium Kön.

- 8246/1. Dactylostegium Nees.

- 8250 add. Rhaphidosperma Don. Rhaphedospera Wiglit.

- 8257 - Andragraphis Wight.

- 8269 - Dicerocaryon 1)tr.

- 8270 - ? Murex Linn.

- 8280 - Oroxylon Std.

- 8286 - Arrabidia A. Juss.

- 8287 - Anemorhegmia Wst.

- 8297 - Spadothea Dumort.

- 8302 - Crateritecoma Lindl.

- 8307 - Kordellestris W'st.

- 8307a $\alpha-$ Hemilobus Wst.

- 8307a $\beta$ - Dilobus Std.

- 8315 - Incarvillaea Orb.

- 8322 - Trigonocarpaea Std.

- 8332 - Trommsdorffia Itr.

- 8336 - Lysionothus Dtr.

- 8341 - Beola Rafin.

Doroceras Stil.

- 8345 - Monophylleia Wst.

- 8361 - Cyrtandria Dtr.

- 8362 - Whitea Dtr.

- 8363 - Rhinchothecum Dtr. Rhynchotecum Lindl. Rhynchotoechum Bl. ('heilosandra Griff.

- 8386 - Orobanchia Vand.

- 8402 leg. Gesnera Plum. Gesneria Li in n. Pentar a phia Lindl.

- 8411 add. Vanhonttea Lemr.

- 8417 - $f$ ? Diphyma Lindl.

- 8429 - Diastemina Lindl.

- 8451 - Epifegus Std. Leptaminium Std. Mylachne stu.

- 8452 - Phaelypaea P.Browne. Phillipaea Wight.

- 8466 - Epirrhizanthus Wst.
XXXVIII. Petalanthae.

n.8479/1. Macrosiphonia Duby.

- 8486 add. Meadea Std.

- 8491 - Asterolinion Brgn.

- 8499 - Euparaea Std.

- 8501a - Tiraseckia Sweet.

- 8507 - Vallenia J.

- 8508 - a.Conomorphida Miq

b. Aconomorpha Ej.

c. Euconomorpha lij.

- 8510 - c. Cybianthoides Miq.

- 8515 - Merista Banks.

Scleroxylon Std.

? Pechea Arr., Std.

- 8516 - ? Tinus Burm.

- 8523 - Oneostemum Lindl.

- 8530 - Cormus Burn.

Izquierdia Fiz. \& P.

Izquierda Willd.

Isquierdia Poir.

Samaria Wiglit.

- 8532 - Purkingia Lindl.

- 8533 - Baeobothrys Vahl.

- 8535 - Zacintha Arr., Std.

- 8540 - Malaspinea Wist.

- 8541 - Nycterisitium Std.

Nycteristion Wst.

- 8542 - Robertsia Wst.

- 8552 - Madhuca Ham., Gmel.

- 8555 - Phlebolithrys Std.

- 8556 - Labourdonnaea Wst.

- 8563 leg. Oinphalocarpus Spreng.

- 8576 add. Cargyllia std.

- 8582 - Bolena W'st.

Stemmatophysum Std. Stemmasiphum D. G

- 8593 - Cryptostoma Dtr.

\section{Bicornes.}

n.8598 add. Solenisia Std.

- 8607 - Linanthe Dumort.

- 8611 - Lyssanthe D)tr.

- 8643a - Eremiastrun Dtr.

- 8649Aa - Calicodon W'st.

- $8649 \mathrm{Bg}$ - Dasyanthus Wst.

- 8649Dg $\beta$ add. Diphylus Std.

(n. $81 ; 8 \%$ del. Oreanthus Walp.)

n.86.99 add. Gaylussacea Wst.

- 8701k - Vitidaea Dumort.

- 8709 - Menziezia J.

- 8713a - Toutousi Duch.

- 8713c - Viereya Std.

- 8713d - Buramea Wst. 
n.8713e add. Hymenanthus Dtr.

- 8722c - Actinocyclus Klotzsch.

- 8730 - Erythorrhiza Wst.

\section{$\mathrm{XL}$. Discanthae.}

n.8740 add. 1)imitopia $\mathrm{Dtr}$.

- 8747 - Schaenolaena Iindl.

- 8765 - Spanante Std.

- 8767b - Sichizilenia Lindl.

- 8776 - Haquetia Dtr.

- 8779 - Alepida I)umort.

- 8781 - Horschfieldia T'ausch.

- 8791 - Heloscia I)umort.

- 8793 - Ptilinium Wst.

- 8795 - Ptichotis Dumort.

- 8796 - Oliviera Dtr.

- 8798 - Crithamus Wst.

- 8801 - Amni Brgn.

- 8808 - ? Pancaseolus Caesalp.

- 8812a - Saxifragia Herm.

- 8826 - Furnrohria Lindl.

- 8830a - Aenanthe Dumort.

- 8832 - Annesorchiza Std.

- 8838 - Kundamannia Std. Cambderia Wst.

- 8839b leg. Pit u ranthos Viv. Pithuranthos D C.

- 8839/1. Eremocarpus Bunge.

- 8856 add. Schnizleinia Std.

- 8880 - Thyselinum Wst.

- 8883 - Thommasinia Std.

- 8886 - Czernievia Std.

- 8898a - Polycyrtas Wst.

- 8903 - Ifdregea Std.

- 8906 - Lefeburia Lindl.

- 8909 - Anetheum Wight.

- 8916 - Oppoponax Dumort.

- 8920 - Symphyloma Std.

- 8921b - Euheraclium Wst.

- 8931 - Hasselquitia Dumort.

- 8939 - Silex Dumort.

Bradleia Wst.

- 8952 - Melanoselium Dumort. Melanospernum Dtr.

-8955 - ? Staphylium Dumort.

- 8956 - Duriaea Lindl.

- 8961 - Szovitzia Wst.

- 8970 - Centhriscus Spr., Std.

- 8972 - Chaerefolium All. Choerophyllum Brgn.

- 8977 - Moloppospermum Dumort.

Molospermum Std.

- 8991 - Anisocladium J)tr. Aniosciadium Lindl.

- 8993 - Pyenocyclus Wst. n 8998 add. Cachris Dtr.

- 9004 - Magydoris Dtr.

- 9017 - Physophora Hfnisg. \& L.

- 9018 - Opodia Wst.

- 9019 - Ceramacarpus Wst.

- 9026a leg. Eumuseniuin W a 1 p.

- 9035 add. Astrema Dtr.

- 9042/1. Heteroptera Marg. \& R.

- 9044 add. Mormoraphis Jack.

- 9044b leg. Oplopanax 'Torr. \& $G$.

- 9045 add. Gussonia Dtr.

- 9052 - 'Torricellia Lindl.

- 9057b - Calyptrophora Wst.

- 9085 - ? Reinera Dennst.

- 9088 - Laea Brgn.

- 9089/1 ? Adenopetalum Turcz.

- 9096 add. Cynoxylum Pluk.

- 9103 - Myzodendrum Forst. Miscodendrum Std.

- 9105 - Razumovskia Wst.

- 9114f - Mocquinia Std.

- 9114n - Spirostyles Dumort.

- 9114 p leg.L e p o stegeres Blume. Lepeostegeres Endl.

- 9120 aild. Dasys Leinr.

- 9125 - Amamelis Lemr. Homomelis Wst.

- 9128 - Foterghillia Dumort.

- 9134 - Staafia Wst.

- 9146 - Ophiria Lindl.

\section{Corniculatae.}

n.9147 add. Buliarda Dumort.

- 9151c - Tetraphylle Lemr

- 9162 - Pistorina Dumort.

- 9165b - Pistorinoides 1)tr.

- 9168 - Aithalis Orb.

- 9171 - Sedum Adans.

- 9174 - Dimorpha Dtr.

- 9175 - Pentorobum Beckm.

- 9179 - Eremosyce Std.

- 9182 - Nimmonia (W.?) Std.

- 9186b leg. Aizonia T'ausch.

Aizoonia Ser.

- 9186d - Dactylites Tausch. Dactyloides Ser.

- $9186 f$ arld. Erophoron Wst.

- 9186h leg. Arabisa Taussh. Arabidia Ser.

- 9191 add. Tolmiaea D G. Tolmica A. Juss.

- 9203 - Callicomis Wst.

- 9233 - Stereoxylum Presl.

- 9235 - Desforgia Std. 
Polycarpịcae, Rhoeades, Nelumbia, Parietales.

\section{Polycarpicae.}

n.9248 add. Pereiria Wst.

- 9254 - Bursaia Std.

- 9274 - Wenlandia Dumort. ? Ferrandia Gaudich.

- 9284 - Chondrodendrum Lindl.

- 9293 - Phytocrena Std.

- 9305n - C'nema Wst.

- 9307 - Bopowia Std.

- 9312 - Sarcopetalum Zoll.

- 9317 leg. Xylopicrum P. Browne. Xylopicron Adans. add. Bulliardia Wst.

- 9319 - Patornia Std.

- 9322 - Porcella 1)tr.

- 9326 - Hexabolus Std.

- 9327 - Anaxagoraea Mart.

- 9342 leg. Sphaeı ostemma R chb.

- 9350 - Sampa c ca Rumph.

- 9352 add. T'asmania Wst.

- 9361 - Reiffenscheidia Std.

- 9362 lcg. Syalita R heede.

- 9363 add. Normbia Dtr.

- 9385 - Trachystella Std.

- 9390 - Narvelia Link.

- 9391 - Cyrtorrhyncha Wst.

- 9392 - T'alictrum All.

- 9392b - Physocarpus Wst.

- 9395d - Petasodes Spreng.

- 9406a - Hecatounia Poir.

- 9417 lcg. Franthus D) umort.

- 9419 add. Gontarella Gilib.

- 9419c - Enemium Std

- 9429 - Actea Dumort.

- 9432 - Actinophora A. Juss.

- 9434 - Xantorrhiza Itr.

- 9443 - Diphyllea Dtr.

- 9445b - Gymmospermum Wst.

- 9449b - Odostemum Std.

- 9450 - Nandiı Kämpf.

- 9450/1. ? Possura Aubl., Std.

\section{Rhoeades.}

n.9459 add. Cerasites Std.

- 9468 - Escholtzia Dumort.

Elschotzia Brgn.

- 9473 - Platystemum Wst.

- 9479a - Eucapnus Wst.

- 9479b - Biscueullata Wst.

- 9480 - biscuculla Wst.

- 9482 - Pistolochia Beruh.

- 9482d - Bulbocapnus Wst.

- 9497 - Nothoceras Dtr.

. 9500 = Cheirantus All. pt.
Cheirianthus Dumort.

n.9502 add. Oudeneya Dtr.

- 9503 - Nasturium Dunnort.

- 9503b - Chrysoraphanus Stu.

- 9519 leg. Leawenworthia Dtr.

- 9527 add. Menioccus Std.

- 9529 - Aubretia 1)tr.

- 9532c - Odontostenum Std.

- 9540 - Petrocalis Dumort.

- 9541a - Veronicella DC.

- 9576 - Ornithorynchium

Röhl.

- 9584 - Dontostemum Std.

- 9586 - Sisymbryum Dumort.

- $9586 \mathrm{~h}$ - Halimolobus Wst.

- 9589 - T'ropidocarpon Dtr.

- $9590 \mathrm{c}$ - Cheirina Dumort.

- 9598 - Leptalium Sweet

- 9599 - Christotelea Dtr.

- 9620/1. Pastorea Tod.

- .9630 add Ethionema Brgn.

- 9632 - Tetrapterigium Dtr.

- 9633 - Isadis Batsch.

- 9638 - Nacrospermum S!d.

- 9646 - Spirorrhyncnus Wst.

- 9654 - Arthrolobos Wst.

- 96tilb - Hebcsinapis DC.

- 9663 - Doupea Dtr.

- 9665 - Origophragmus Dtr.

- 9667 - Dyplotaxis DC.

- 9667a - Cátocarpaea DC.

- 9067b - Anocarpaea DC.

- 9675 - Carichtera Dumort.

- 9685 - Arthrobolus Wst.

- 9687 - Didesmis Loud.

- 9724 - Thylaucium Dtr.

- 9726 - Podoriocarpos Lam., Std.

Podoriocarpus Wst.

- 9733 leg. Moris o 11 a Plum. Morisonia Linn.

- 9734 add. Apioscorodon Pluk.

- 9735 - Ritcheia Brgn.

- 9736 - Stephanium Juss.

- 9747 - Xanthoreseda G.Don:

\section{Nelumbia.}

n.9761 add. Sarraziuia Hfmsg. Sarracinia Wst.

- 9766 - Tanara Roxb.

\section{Parietales.}

11.9768 add. Cisthus Beckun. Ladanum Tausch.

- 9768a - Chrysorhodium Std.

- 9768a $\beta$ - Leucorhodium Std. 
n.9770 add. Ledon Tausch.

- 9770a - Cyticystus Burm.

- 9780 - ? Ireum Burm. (Std.)

- 9782f - Herpetium Wst.

- 9783a - Hibanthus Dtr.

- 9785 - Noisettea Dumort.

- 9788 - Corinostylis A. Jus:.

- 9789 - Braddleya Std.

- 9798 - Tachytes Std.

- 9806 - Fouguiera Dtr.

- 9809/1 ? Stachycrater Turcz.

- 9811 add. Orleana Herm.

- 9818 - Eriudaphos Wst.

- 9825 - Fonara Dtr.

- 9829 - Christiannia Wst.

- 9830 leg. Da sianthera Presl. Irasyanthera Rehb.

- 9847 add. Hydrocarpus Itr.

- 9850 - Marshallia Gmel.

- 9851 - Asteranthus Std.

- 9861 - Ryana Wst.

- 9862 - Bülovia Wst.

- 9867b $\delta$ leg. Polyanthema G. D on.

- 9872c add. Distephania Std.

- 9880 - Physema Rchb.

- 9888 leg. Menzelia J، in 1.

- 9888 b add. Creolybus Wst.

- 9895a - Helicterioides Std.

- 9904 - Papaja Rumph.

- 9905 - Vasconcellea Planch.

\section{Peponiferae.}

n.9925 add. Bryonea Wight.

- 9930 leg. Ecballium I) u mort.

- 9933 add. Beninkasa Std.

- 9960 - Erythroropalum

Blume.

- 9964 - ? Herpetospermum Wall., Std.

- 9976 - ? Falkea Kön.

- 9991 leg. Diploclinium Iindl.

\section{XL.VII. Opuntiae.}

n.10017a leg. Eu m a millaria Eng. Eumammillaria Míill.

- 10017b leg. Coryph an th a Eng. Coryphanta Müll.

- 10037 add. Peiresǩia Stil. Carpophyllus Neck. Carpophyllum Std.

\section{Caryophyllinae.}

n.10047 add. Diploclıonia Std.

- 10061 - Calandarium ${ }_{n}$ Juss.", Std. n.10068 add. Leptotrina Wst.

- 10075 - Psammotrophia Meisn.

- 10078 - Schieda A. Juss.

- 10084 - Levisia Std.

- 10096 - Louichia Std.

- 10102 - Cordia Rchb.

- 10109 - Delia Dumort.

- 10117 - Buffonea Kuch.

- 10137 - Larbraea Dumort.

- 10139/1. Myosanthus Dumort.

- 10141 add. 1)yanthus P. Browie.

- 10160 leg. Lychnanthus ( $\mathrm{mel}$. add. ? Mönchia Neck.

- 10173 - Nicrothea A. Juss.

- 10179 - Phitolacca All.

- 10180 - Ericilla Std.

- 10185 - Gyrostemum Std.

\section{Columniferae.}

11.10190 add. Kitaibella Reuss.

- 10200c - Lebretonnia Brgn.

- 10205 - Hybiscus Dumort. Kosteletskya Prgn。

- 10205/1 ?Arcynosperinum Turcz.

- 10215 leg. Gossipium Herm.

- 10217/1. Julostylis Thwait.

- 10237/1. Cullenia Wight.

- 10240 add. Ochrosma Dumort.

- 10241 - Cheirostemum Std.

- 10248 - Asterotrichium Wst.

- $10254 \mathrm{c}$ - Alicterus Wst.

- $10259 \mathrm{c} \beta$ - Astrodendron Wist.

- 10263 - Pterigota A. Juss.

- 10270/1 ? Sczegleewia Turcz. Szeglewia Müll.

- 10279 add. Kerandrenia Std.

- 10281/1 ? Peltostegia Turcz.

- 10282 add. Rulingea Dumort.

- 10288 - ? Butneria P.Browne.

- 1029:3 - Kleinhowia Brgn.

- 10296 - Malvinda Burm. Lopanthus Vitnı, Std.

- 10298 - Riedlia Dumort.

- 10303/1. Physocodon Turcz

- $10310 \mathrm{~b}$ add. Acropetalum A.Juss.

- 10313 - Glossostemum Std.

- 10315 - Peterospermum Wight.

- 10316/1. Leptony chia .Turcz.

- 10325/1. 1)asycarpus Örst.

- 10335 add. Honckeneya Std.

- 10338b leg. Bartramea I) C.

- 10341 add. Brownolowia Std.

- 10341/1. Diplodiscus Turcz. 
n.10341/2. Pityranthe Thwait.

- 10343ar add. Syphomeris Std.

- 10313a - ? Micrococos Burm. Micrococcus Beckm.

- 10:54 add. Flaiocarpos Burm. Eriostemum Colla.

\section{Guttiferae.}

n.10369 add. Saul Roxb.

- 10377 - T'antalus Nor.

- 10379 - Schisolaena Pet.-Th.

- 10381 - Pellinia Molin.

- 10392 leg. Furia Camb.

- 10397 add. Lettosomia Dumort.

- 10398 - Saurauia Wst.

- 10408 - Martinieria Std.

- 10409/1 ? Tristylium Turcz.

- 10412/1. Hypopogon 'T'urcz.

- 10425 add. Schweiggeria Wst.

- 10484 - Blackstonia A.Juss.

- 10435 leg. Mamei Plu m.

- 10437 add.Xanthochysmus Dum.

- 10443 - Callophyllos Beckin. leg. Inophyllum Burm. Bint a ngor Rumph. Calaba Plu un.

- 10450 add. Machanaea Std.

- 10455 leg. Ascyum DC. (non Vahl).

add. Noranthea Dumort.

- 10458 leg. Hypericoides P l um. add. Knifa Adans. pt.

- 10459 - Triadenoides Walp.

- 104591 - Noryska Std.

- 10471 - Reaumurea Std. ? Beaumulix Willd.

\section{Hesperides.}

n.10480 add. Verniseckia Std. Wernisechia Wst.

- 10487/1. Poraqueiba Aubl.

Barreria Scop.

Bareria Juss.

Meisteria Gmel.

- 10498 add. Cassiera Räusch.

- 105(13c - Spermaxyron Std.

- 10505a - Codonia Wst.

- 10510 - Anacolosia Wst.

- 10522 - Glyscosmis Dtr.

- 10526 - Nimbo Dennst.

- 10529 - Clausenia Dumort.

- 10530 - Micromelon Std.

- 10531/1. Arthromischius 'Thwait.

- 10534 add. Aglaia Dumort. Belon Wst.

- 10535 - Limo Rumph.
Limonellus Ej.

Sarcodactil is Gärtn. Sarcodactylis Std.

n.10537 add. Chinotria Dtr.

- 10554 - Lanisiuin A. Juss.

- 10556 - Aphanomixis Wst.

- 10556/1 ? Oraoma Turcz.

- 10558 add. Chisogeton Std. Chizocheton A. Juss.

- 10500 - Hartigsea Std. Hartigsia IVst.

- 10562 - Cabralia Wst.

- 10569 - Trichila Wst.

- 10570/1 ? Dasycoleum 'Iurcz.

- 10571 add. Guaria Dumort.

- 10586b - ? Cuveraca Jon.

- 10586/1 ? Piptosaccos T'urcz.

\section{Acera.}

n.10592 add.c. Eucamarea Griseb. d. Chamaea Ej.

- 10595 - Gaudichautia St. Hil. Gandichaudia Dum.

- 10595a .- Tritomopteris Wst.

- 10596 - Aspicaria Dtr.

- 10600 - I)iplopteris Griseb.

- 10601 del. b. Mascagnia Bert.

- 10606/1. Mascagnia Bert. Mascagna Std.

a. Notopteris Griseb.

b. Pleuropteris Ej.

c. Eumascagnia Ej.

- 10607 add. [a. Eutetrapteris Griseb.

b. Pentapteris Ej.

c. Stauropteris Ej.

d. Schizopteris Ej.]

- 10607/1 ? Stenocalyx Turcz.

- 10608 add.[a.HolopetalonGriseb.

b.PtycheteropterisEj. $\alpha$. Stenotorus Ej.

c. Euheteropteris Ej.]

- 10512 - [a.Hemiramma Griseb. b. Eubanisteria Ej.]

- 10613 - Rhyssopterys Wst.

- 10614 - Sigmatophyllum Dtr. Stigmaphyllum Wst.

- 10618 - Malpigia P. Browne.

- 10623a - Euburdachia Griseb.

- 10629/1. Clonodia Griseb.

- 10630/1. Diacidia Griseb.

- 10630/2. Glandonia Griseb.

- 10634/1 ? Stenocalyx 'Turcz.

- 10637 leg. Erythroxylum P.

B rowne.

Erithroxylum Juss. 
n.10641 add. Halicacabum J.Bauh. Cor Indum Herm., Burm.

- 10646/1 ? Lophozonia Turcz.

- 10649 add. Schmidelea Wight.

- 10652 - Valenzuela Std.

- 10662 - Bligia Dumort. Dimeresia A. Juss.

- 10673 - Laetji Osb.

- 10684 - Sleichera A. Juss.

- 10699 leg. Dodonea P.Browne.

- 10704 add. Phaeocarpos Std.

- 10715 - Cupanioides Brgn.

\section{Polygalinae.}

n.10740 add. c. Pterisma Presl.

- 10745 - Kaulfussia Dennst.

\section{Rhamni.}

n.10747 add. Citriobathus A. Juss. - 10758 - 'Jurinia A. Juss.

- 10763 - Staphilaea All.

- 10767/1. Melanocarya 'Turcz.

- 10769 add. Polycandia Std.

- 10770/1. Cathastruin 'Jurcz.

- 10783/1 ? Cienkowskia Rieg. \& Rach.

- 10789 add. Nerija Std.

- 10797 - C'oupia 1)tr. ? Schranckia Scop.

- 10802 - Custenia Std.

- 10803 - Anthodiscus (Endl.) Wst.

- 10808/1. Prinodia Griseb.

- 10821 add. Jujuba Burm. Mansana Gimel.

- 10828 - Scutea Wight.

- 10829 - Vittmania Wight.

- 10842 - Tricocephalus A. Juss.

- 10856 - Govana All.

- 10859 - Solenanthe Wst.

- 10860/1 ? Tetrasperma G. Don.

\section{Tricoccae.}

n.10877 add. Euphorbio-Tithymalus Burm. Ceraselma Wst.

- 10884 - DalechanısiaWight.

- 10894 - Exoecaria Dumort.

- 10903 - Synapisma Std.

- 10907 - Homalanthes Std.

- 10909 - Falconera Wight.

- 10917 -?Chamaelaea Burm. pt.

- 10923 - Leptorrhachis Wist.

- 10927 - Pluckenetia Std. n.10959 add. Cladogynus Wst.

- 10978 - Carcia Räusch.

- 10992 - Driandra Corr. Elaeococcus Std. Elaeococcos Wst.

- 10993 - AdenorrhopiumWst.

- 10994 - Bromfieldia Wst.

- 10994b - Mozina Brgn.

- $11001 \mathrm{leg}$. Ricinocarpos Ma r ch.

- 11024 add. Leptomon Std.

- 11029 - ?Ricinokarpos Burm. Schradera Willd. leg. Elutheria Linn.

- 11060 add. Argyrothamnia Wst.

- 11062 - Trigonostemum Wst.

- 11068i1. Calpigyne Blume.

- 111169/1. Pycnosandra Plume.

- 11088 add. Pierardea Wight.

- 11093 - Phillanthus Allion.

- 11093/1. Chorisandra Wight.

- 11099 add Kirganellia llumort.

- 11102 - Frankia Berter.

- 11107 - Glochidinopsis Std.

- 11108 - Glochidium Wst. Bradleja Gärtn.

- 11125 - Securigena Dumort.

- 11142 - Aporusa Blune.

- 11148 - Mebora Std. Taphranthus Wst.

- 11167/1. Bucheria Wst.

- 11168 add. Desfontaina Std.

- 11171/1. Orbignia Bert.

- 11172 leg. Antidesma B u rm.

- 11174/1? Renardia Turcz.

\section{Terebinthinae.}

n.11175 add. Scorias Wst.

- 11188 - Brasiliastrum Lam. pt.

- 11198 - Litria Hook.. Std.

- 11199c - ? Philostemon Raf. Philostemum Std.

- 11201 - Laurophillus Röm. \& Sch.

- 11223 leg. Mo n bin Pium. Nombin DC.

- 11239 - Bursera Linn. Burseria J a c q.

- 11239/1 ? Sarcotheca Turcz

- 11240 add. Trattinickya A. Juss.

- 11252 - Tapinia Std.

- 11254 - Triceras Wst.

- 11255 leg. Barb i lus P. Browne. Barbylus Juss.

- 11270 add. ? Chamaelaea Burm.

- 11272 - Luxenburgia Stud.

- 11276/1 ? Charidion Bong. 
n. 11283 add. Vittmannia Dietr. Manduyta Std.

- 11288/1 ? Oligostemon 'Turez.

- 11304 add. Fagaras Burm.

- $11304 b$ - ?Lacuris Ham., Sid.

- 11304d - Ochroxylon Std.

- 1131)4e - Macqneria Kunth.

- 11310 - Rubentia Boj.

- 11312 - Roscia 1)tr.

- 11313 - Ptelia Dir.

- 11324 - Ściurns I)tr. Dangervillea Arr., Std.

- 11329 - Ertelia Std.

- 11343 - Ziera Lumort.

- 11352 - Choribena Std.

- 11356 - Corraea Sm.?, Peuss. Mazentoxeron W'st.

? Euphocarpus Anders.

- 11366 - a. verae Bartl. \& W. b. Trichopodes Eor. c. Agathosmoides Eor.

- 11367 - Agasthosma Brgn.

- 11388/1 ? Systemon Reg.

- 11385 add. Heterozyges Wst.

- 11389 - Röperia Std.

- 11398 - Gayacum Brgn.

- 11399 - Pegamum Brgn.

\section{Gruinales.}

n.11407a add. Grenwillea Std.

- 11407k - 1)ibrachia Std.

- 11410 - Cruickshanksia Std.

- 11414 - Clethra Bertero.

- 11420 - Sassia Molin.

\section{Calyciflorae.}

n.11440b add. Mirobalanus Std. Badannia Wst.

- 11443 - Petraea Ung.

- 11455 - Cacoutia Std. Hambergeria Wst.

- 11464 - Rizophora Burm. ?

- 11464a leg. Mangles Plum. Mangle Adans.

- 11470 - Baraultia Spreng.

- 11484a add. 1)anthia Stil.

- 11489d - Pleurostemum Wst.

- 11496a leg. Chamaenerion G r a y.

- 11497 add. Zauscheria Std.

- 11501 - Macrostemma Sweel.

- 11514a - Pentapterophyllum Wst.

- 11516 - Proserpinaea Orb.

- 11524 leg. Pankea Örst. Panke Feuill. n.11535 add. Hypobrychia Wst.

- 11540 leg. Ammannella Miq.

- 11541 add. Nessea Std.

- 11541b - Chrysolyga Wst.

- 11544a - Amannoides Dtr.

- 11550 - (iriselea Dtr.

- 11555 - Diplosudon I)umort.

- 11556 - Calycoplectus Wst.

- 11558 - e. Rytidotrochis Blume.

- 11568/1. Macclellandia Wight.

\section{Myrtiflorae.}

n.1157: add. Jaraphaea Std.

- 11574c - Erioloma Wst.

- 11583 - Comollia Wst.

- 11594 - b ? Stomandra Jack.

- 11600 - Jonacenta Std.

- 11605 - Othanthera Std.

- 11607 - Plerolepis A. Juss.

- 11609 - Osbeekia Wight.

- 11617 - Chaetagastra Crüg.

- 11635b - Calorrhexia Wst.

- 11644 - Diplogenaea A.Juss

- 116ti2 - Salpingea I'tr.

- 11680a - Tetrastemum Wst.

- 11681)b - Octostenium Sta. Octostomon Ej.

- 11682 - Calygonium Dtr.

- 11692 - Apatilia Rehb.

- 11694 - Decaraphe A. Juss. Decarrhaphe Wst.

- 11694b - Gallasia Mart.

- 11697a - Stephanothricum A. Jus?.

- 11717 - Gräffenrieda Dtr

- 11720 - Axinea Juss.

- 11739 - Cornus Burm.

- 11749 - Marliera Dtr.

- 11750 del. Zuzygium Fj.

leg. Suzygium P. Hrowne. Chytralia Adans.

- 11760 add. Suarda Nocca. Olythia Std.

- 11770 - Syzigium Std. Sizygium Puch. Zyzygium Brgn.

- 11773 - Jannbos Burm.

- 11783 - Pela liheede. Cujavus Rumph. Guajavos Burm.

- 11803/1. Paivaea Merg.

- 11806 add. Feliciana Wst. Tetrastemum Wst.

- 11809b - Merichea Wst.

- 11812 - ? Cumbea Buch 
n.11813 add. Teichmeyera Wst.

- 11814 leg. Courataria Du mort.

- 11816 add. a. Eulecythis Berg.

- 11818 - Bertholetia Brgı.

- 11820 - Granata malus Burm.

- 11828 - Chamaelancium Dum.

- 11839 - Bäckia R. Br., Nees.

- 11862/1 ? Stenospermum Sweet.

\section{Rosiflorae.}

n.11893a add. Apyrophorum W'st.

- 11893f - Sobrus Reich.

- 11893g - Adenorrhachis Wst.

- 11904 - Mespylus All. pt.

- 11905 - Stransvaesia A.Juss.

- 11910 - Hulthenia Brgn.

- 11915 - Fraga Lapeyr.

Lehinannia Tratt.

- 11915b $\beta$ - Geoides Tausch.

Lupinoides Ej.

- 11921 - Spalanzania Dtr.

- 11933 - Margyriocarpus Dtr.

- 11941 leg. Or e ogeum Ser.

- 11943a add. Pryados Tausch.

- 11951 - Spirea Pall.

- 11951a - Icoturus Wst.

- 11951e - Eriogyne Std.

- 11954 - Gillonia A. Juss.

- 11958 - Kagenackia Std.

- 11963 - Nevreda Dtr.

- 11968 - Cerasoides Wst.

- 11969/1 ? Eriolithis Gärtn.

- 11971 add. Hirtellia Dumort.

- 11978a - ? Ferolia Aubl.

- 11982 - I.ecostomum Std.

- 11985 - Trilipsium Std.

\section{Leguminosae.}

n.11991 add. Paptisia Dtr.

- 11992b - Ibbertsonia Std.

- 12004 - Chorosema Brgn.

- 12013 - Hugelroea Sitd.

- 12022 - Pultaenea Brgn.

- 12033 - Physicarpus Wst.

- 12037 - Possieva Std.

- 12039 - Tempeltonia Std.

- 12048e - ? Crypsocalyx A. Rich.

- 12053 - Harpeloma Wst. Hosakia Grah.

- 12056d - Calobotra Std.

- 12110 - Dorychnium Brgn.

- 12125 - Poecadenia IVst.

- 12131 - Astragalus Burm.

- 12133 - Glychirriza All.

- 12147 - Brongnartia Std. n.12158 leg. Emerus B u r m.

- 12164 add. Corynites Wst.

- 12183 leg. Guldenstädtia

D um ort.

- 12187 add. Oxytropus Gray.

- 12197d - Sissolia Rupp.

- 12211 - Ormacarpum Wight.

- 12218 - Trifolium Burm.

- 12221 - Chapmania Wst.

- 12224 leg. Aeschinomene All.

- 12239 add. Unobrychis Burm.

- 12270 - Flos clitorius Burm.

- $12274 \mathrm{lg}$. P le c tr o tropis Schum.

- 12279 add. Zichia Std.

- 12280 - Physalobium Std.

- 12282 - Kennedynella Std.

- 12290 - Sliutera Wight.

- 12291 - Campesia Wight \&A. Galactea Wight.

- 12303a - Colchitropis Std.

- 12303b - Eucannavalia Std.

- 12305b - Strizolobium Wst.

- 12309b - Nauricon Wst.

- 12325 - Sphenostyles Std.

- 12345c - Moghamia Std.

- 12346 - Orobus Burm.

- 12364 - Pterocarpus Berg.

- 12366 - Clypeola Burn. Peltaria Ej.

- 12366/1 ? Hymenolobium Benth.

- 12381 add. Dahlbergia Vahl.

- 12383 - Semeionitis Std.

- 12384 - Mischolobium Wst.

- 12389 - Callisema Std.

- 12393 leg. Geoffrosa Ja c. q. Geoffraea L in $n$.

- 12399a add. Cumarouma Std. Coumarouma Wst.

- 12406 add. Coublandea Std.

- 12408 - Edwarsia Dumort.

- 12413 - Calpurnea Std.

- 12417 - Cladrastris Std.

- 12427 - Tesota A. Gr., Müll.

- 12438 -Parkensonia P.Browne.

- 12441 leg. GuilandiaP.Brow ne. add. Guillandina All.

- 12442 - ? Crista pavonis Breyn, Burm.

Poinciniana Duch.

- 12445 leg. Ca esalpina Plum. Caesalpinia Gled., Linn.

- 12454 add. Colvilleia Std.

- 12456 - Cladotrichum Std.

- 12460 - Moldenhaweria Std. Dolichonemia Std. 
n.12461g add. Lasiorrhegma Wst.

- 12466 leg. Zollernia Nees. Acidandra Mart.

- 12467a add. Gynanthistrophe Std.

- 12473 leg. B row n a e a Jacq. Brownea Linn.

- 12476 add. Heterostemum Std.

- 12480 - Humboldia Rehb.

- 12481 - Schottia Brgn.

- 12493 leg. Anthonotha S pren g.

- 12500 add. Valcarcella Std.

- 12505 - Cusparia Dtr.

- 12522 - ? Opalatoa Lam.

- 12544 - Moringia Dtr.

- 12545 - Gledisia All. Caesalpinoides "I.", Std.

- 12566 - Sassa Bruce, Gm.

- 12566a - Racosperma Wst.

- 12578 - Pithecollobium Std. Pithecellobium Wst.

- 12584 - Clomopanus Std.

\section{Appendix.}

n.12624 vid. 3097/1.

- 12635/1. Anisophyllum Jacq.

- 12637 add. Styxis Dtr.

- 12645 - Barbevia Std.

- 12652/1. Brabila P. Browne.

- 12658/1. Calvaria Gärtn. f. Clavaria Std.

- 12665/1. Cistellaria Schott.

- 12668 vid. $4686 \mathrm{~d}$.

- 12668/1. Collococcus P. Browne.

- 12668/2. Cominia P. Browne.

- 12683/1. Echyrospermum Schott.

- 12683/2. Elemifera Linn. Toxicodendrum Cat.

- 12687 vid. 7904.

- 12688 add. Falopia Wst.

- 12689 vid. 11978a.

- 12690 - 7488 .

- 12691 - 7507.

- 12694 - 6746.

- 12695/1. Halimus P. Browne.

- 12695/2. Havannella Iinn.

- 12700 vid. 8530.

- 12706/1. Leciscium Gärtn. f.

- 12711 vid. 8552.

- 12711/1. Malacoxylon Jacq.

- 12711/2. Malicope Vitm.

- 12713 vid. 10821.

- 12714 - 9850 . n.12717 vid. $10487 / 1$.

- 12718 - 6956.

- 12719/1. Melanthus Weig.

- 12723 vid. 10160.

-12726 - 8270 .

- 12729 - 10526 .

- 12732/1. Ocymophyllum Buxb.

- 12732/2. Orectospermum Schott.

- 12735 vid. 8386.

- 12737 - 4901 .

- 12741 - 7859 .

- 12741/1. Pectinea Gärtn.

- 12744 vid. 11199c.

- 12747/1. Piperodendron Heist.

- 12750 vid. $10487 / 1$.

- 12752 add. Pseudobasilicum Std.

- 12755/1. Pyxidaria Schott. Pixidaria Isis.

- 12757 vid. 9085a.

- 12765 - 12566.

- 12766 - 11420 .

- 12768 - 10797 .

- 12769/1. Scobedia Lab., Std.

- 12778 vid. 7582.

- 12780 - 4688.

- 12784/1. Uvifera Pluk., Linn.

- 12789 add. Walkuffa Std.

- 12792 leg. Xyloc ys te P.Browne. Xylociste Adans.

n.12814 vid. 4675 .

n.12834/1. Carlotea Arr.

- 12836/1. Catanga Aubl., Std.

- 12838/1. Cavaria Ruiz \& P.

- 12852/1. Euphorona Arrab.

- 12352/2. Favrodina Reyn.

- 12868 vid. 4883.

- 12874/1. Meleagrinex Arr., Std.

- 12875/1. Michauxia Räusch.

- 12883/1. Oxyglycus Bowd.

- 12887/1. Petrotheca Rudge.

- 12887/2. Plagiocaryum Willd.

- 12888/1. Pleragina Arrud.

- 12892/1. Pulchia Arrab.

- 12892/2. Raphelingia Dumort.

- 12892/3. Ribeirea Arrud.

- 12897 add. ? Serjana Arrab.

- 12899/1. Skolemora Arrud., Std.

- 12900/1. Sutherlandia Räusch.

- 12901/1. Tasalia Ruiz \& P.

- 12902/1. Tilesia Thunb., Std.

- 12902/2. Tooldia Lehm., Wst.

- 12907/1. Zedoaria Burm. 


\section{Addenda et Corrigenda in Indice alphabetico.}

Abies Brongn. 4554 A.

Abilg a a rdia Poir. 3243.

Achiranthes P. B rowne 4904 .

Achranthes Dumort. 4904.

Achyrobaccharis Sch. Bip. 5542/1.

Aconomorpha Miq. $8508 \mathrm{~b}$.

Acquartia Rc h b. 7822 .

Acrochordodendros Pluk. 6677.

Acropteris Presl 2312b.

Acrospelton Wst. 2906d.

Acrostiche Dtr. 8611.

Acrostolia Dumort. 2092b.

Acrostoma Didr. 6873/1.

Actea Dumort. 9429.

Actinocyclus Klotzsch 8722e.

Adenocarpus Wst. 5743.

Adenopetalum Turcz. 9089/1.

Adenorrhachis Wst. 11893g.

Adenorrhopium Wst. 10993.

Adenosachma A. Juss. 6915.

Aeceolades Wight 4044.

Aenanthe Dumort. 8830a.

Aërophyton W'st. 197.

Aeschinomene A 11. 12224.

Agallostachys Beer 3758/1.

Agasthosnia Brgn. 11367.

Agathosmoides B. \& W. 11366 c.

Aggeianthus Wight 3852/2.

Aglaia Dumort. 10534 .

Aizonia Tausch $9186 \mathrm{~b}$.

Aizoonia Ser. $9186 \mathrm{~b}$.

Alephia Dumort. 3250.

Alepida Dumort. 8779.

Alicterus Wst. 10254c.

Alonsea Dtr. 7909.

Alonzoa Brgn. 7909.

Alpina Plum. 4265.

Alpinia Gärtn. 4265 .

Alveolaria Sternb. 2633 (b).

Amannoiảes Dtr. 11544a.

Amaranthoides Burm. 4881.

Amarocarpus Wst. 6733.

Amblacheniuin Wst. 6394.

Amblirium Wst. 3414a.

Amblyogonum Wst. 4949 d.

Amni Brongn. 8801.

Amoleiachyris Walp. 5469:

Ampalus Wst. 4694.

Amphirhaphis Walp. 5492.

Amphir h a p is DC. 5492.

Amphirrhapis Wst, 5492.,
Amphirrhinum Wst. 2200 D.

Amphochaeta Anders. 2770/1.

Anacheilum Wst. 3869 .

Anacolosia Wst. 10510.

Ananthocyclus Wst. 5951 .

Anaphalanthus Wst. 6233c.

Anaster Dumort. 7045.

Anaxagoraea Mart. 9327.

Anclyanthus A. Juss. 6774.

Andragraphis Wight 8257.

Andrapogon Roy. 3084.

Andriana Brongn. 2546.

Andropogum Brongn. 3084.

Aneseia Wight 7754.

Anetheum Wight 8909.

Anisocladium Dietr. 8991 .

Anisophyllum Jacq. 12635/1.

Anleya A. Juss. 8049.

Annesorchiza Std. 8832.

Anocarpraea DC. $9667 \mathrm{~b}$.

Antheeis chima Korth. 10414b.

Anthelmenthica P. Br. 7040.

Antherosperma Poir. 4997.

An the rotoma Naud. $11609 \mathrm{~b}$.

Ant he rotriche Turcz 10351.

Anthiaris Duinort. 4731.

Anthodiscus Wst. 10803.

Anthonotha S preng. 12493.

Anthristiria Dumort. 3072.

Anthrostylis Dtr. 3185 .

Antichloa Sweet 2880.

Antidesma B urm. 11172.

Antirrhinum Burın. 6815 .

Apatilia Rchb. 11692.

Apetalon Wight 4239/1.

A phanomixis Wst. 10556.

A pioscorodon Pluk. 9734.

Aplophlebis Brongn. 2314a.

Aplotrichum Dumort. 2270/3.

A pocynophyllum Vis. \& Mass.2313.

Apodanthes Poit. 2693.

A ponogetoa Dumort. 3328.

Aporusa Blume 11142.

Aprica Dtr. 3434a.

Apuleia Less. 6202.

Apuleja Gärtn. 6202.

Apyrophorum Wst. 11893a.

Arabidia S e r. $9186 \mathrm{~h}$.

Arabisa Tausch $9186 \mathrm{~h}$.

Arachnoides W st. 2529.

Arcynospermum Turcz. 10205/1. 
Argyranthes Wst. 6099.

Argyrochaita Wst. 5668c.

Argyrophytum Wst. 5845.

Argyrothamnia Wst. 11060.

Ariscema Std. 4:33.

Aristotelia Wst. 4163a.

Aro-Orchis Burm. 4259.

Arrhostoxyl on Mart. 8142.

Arrhostoxylun Nees 8142.

Arthrobolus Wst. 9685.

Arthrochloa R. Brown 3096/1.

Arthrolobos Wst. 9654.

Arthromischius Tliwait. 10531/1.

Arthronia J) umort. 949.

Arundenia Wight 3894.

Asagraya Wst. 3369 .

Asclepias Rebent. 7264.

Ascochilus Blume 3972.

Ascyum DC. (non Vahl) 10455.

Aspicaria Dtr. 10596.

Aspidium Nath. 2487.

Asplenieae p. 61 .

Asplenium Sternb. 4653c.

Asteranthus Wst. 9851.

Asterolinion Brongn. 8491.

Asterothria Grell. 6405.

Asterotrichium Wst. 10248.

Asterotrix Brongn. 6405.

Astragalus Burm. 12131.

Astrema Dtr. 9035.

Astrocephalus Dumort. 5234b.

Astrodendron Wst. $10259 \mathrm{c} \beta$.

Astropolium Nutt. 5384.

Athenantia Dumort. 3096.

Atherophora Willd. 2837.

Athyrium Math. 2486.

Atractus Kirby 1685/1.

Atraphraxis Brongn. 4945.

Atrichium Wst. 2193a.

Aubretia Dtr. 9529.

Aulacomnium ]3ayrh. 2186b.

Aulacorrhynchus Wst. 3127.

Aulaxis Std. 2761.

Auleya Dtr. 8049.

Aulonemia Goud. 2996.

Axinea Juss. 11720.

Axinopus Wst. 2748.

Axopus Dumort. 2748.

Babounga Wst. 5894b.

Bacopia A. Juss. 7981.

Badannia Wst. 11440 b.

Bäckia R. Brown, Nees 11839.

Baeobothrys Vahl 8533.

Baeothryum Std. 3229b.

Baldellia Parl. 3330/1.

Balenerdia Std. 5071 .
Balınisia Std. 4332.

Balotte Neck. 7523.

Bambusoides Wall. 3002/1.

Baraultia Spreng. 11470.

Barbevia Std. 12645.

Barbilu s P. Browne 11255.

Barbylus J us s. 11255.

Barckhansenia Menke 6455 .

Bardanesia Dumort. 6275.

Bareria Juss. 10487/1.

Barkausia Brgn. 6455 .

Barkeria Knowl. \& W. 3884.

Barreria Scop. 10487/1.

Bartholinia Dumort. 4112.

Bartramea DC. 10338b. (non Gärtn.)

Basilium Wst. 7426.

Batracliosperma Bory 471.

Baziasa Std. 5611.

Beaumulix Willd. 10471.

Beckera Nees 2771/1.

Beckeropsis Fig. \& Not. 2771/1.

Becquerela Nees 3149.

Becquerelia Brong n. 3149.

Bellidaster Dumort. 5381 .

Belon Wst. 10534.

Bembicodium Wst. 5962.

Benedicta Wst. 6210b.

Benincasa Savi 9933.

Benincassa Wight 9933.

Beninkasa Std. 9933.

Bennetia Rafin. 2830.

Std. 6210.

Beola Rafin. 8341.

Berghias Std. 6944c.

Beriesa Std. 4877.

Berliera Std. 6978.

Bertholetia Brongn. 11818.

Bertiera Hamilt. 6978.

Besha Dtr. 3000.

Bibliolithes Schloth. 4320.

Bilbergia D u mort. 3761 .

Bint ang or Rumph. 10443.

Bipennula Std. 4227.

Biscuculla Wst. 9480.

Biscucullata Wst. 9479b.

Blainvillia Wst. 5606.

Bleekrodea Blunie 4697/1.

Bligia Dumort. 10662.

Blosvillea Decne 572.

Boeberoides Std. 5788c.

Bohmeria Dumort. 4763.

Bojera Std. 5297.

Bolena Wst. 8582.

Bonara Dtr. 9825.

Bonifacia Manso 6855. 
Bonneya Dtr. 7993.

Boophana Std. 3707.

Bopowia Std. 9307.

Bosaea Dumort. 4810.

Bosia L inn. 4810.

Bossieva Std. 12307.

Bothryospermurn Std. 7703.

Boutelouoa Wst. 2880.

Boutonia Ht. Erford. 6541.

Bowea Std. 7968.

Bowringia Hook. 2430.

Brabeum Beckm. 5162.

Brabila P. Browne 12652/1.

Brachistelma Dumort. 7338 .

Brachycalymna Wst. 5349b.

Brachycladus Wst. 6301.

Brachycoris Std. 7968.

Brachyrrhainphus Wst. 6436.

Brachyrrhynchus Wst. 6156.

Bradburya Wst. 5477.

Braddleya Std. 9789.

Bradleia Wst. 8939.

Bradleja Gärtn. 11108.

Brasiliastrum Lam. 11188.

Brochidium Perty 423.

Brochinia Std. 3771.

Bromfieldia Wst. 10994.

Broomheadia Wight 3968.

Brongnartia Std. 12147.

Brownaea Ja eq. 12473.

Brownea Linn. 12473.

Brownolowia Std. 10341.

Bruckmannia Nonn. (non Sterub.) 2282.

- Wst. 2729.

Brugmannsia Std. 2690.

Brukmannia Stern b. 2282.

Bryonea Wight 9925.

Bucephalum Std. 4719.

Buchanania Smith 7442 .

Bucheria Wst. 11167/1.

Bucholzia Wst. 6651.

Buchosia Std. 3399.

Bülovia Wst. 9862.

Buffonea Koch 10117.

Bugainvillaea Brongn. 4975.

Bulbocapnus Wst. 9482d.

Buliarda Dumort. 9147b.

Bulliardia Wst. 1670. 9317.

Bumeloides Std. $7004 \mathrm{~b} \alpha$.

Buramea Wst. 8713d.

Bursaia Std. 9254.

Bursera Lin n. 11239.

Burseria Jac q. 11239.

Butneria P. Browne 10288.

Cabralia Wst. 10562.
Cacalia Burm. 5252h.

Cacaliastrum Std. 6150a.

Cachris Dtr. 8998.

Cacoutia Std. 11455.

Cadelari Adans. 4904c.

Cadelium Medic. 12321.

Caenogium Lindl. 1067/1.

Caesalpina Plu m. 12445.

Caesalpinia Gled., Linn. 12445.

Caesalpinoides "L." Std. 12545.

Caesulea Wight 5591.

Caeta Std. 7988.

Caetocapnia Endl., Std. 3755.

Caidbeia Std. 4775.

Caidbeja Forsk. 4775

Calaba Pl u m. 10443.

Calaena Li nd 1. 4217.

Calamites Sternb. 2633 (d).

Calamodendron Brgn. 2282/1.

Calandarium Std. 10061.

Caleacte R. Brown 5851c. - Less. 5847.

Calendria Reuss 6169.

Calicodon Wst. 8649 Aa.

Calinux Std. 5063.

Calista Ritg. 4245.

Callestephus Wight 5409.

Callichloë Willd. 3084 .

Callicodon Benth. 8649 Aa.

Callicomis Wst. 9203.

Callimeris Spach 5390.

Callisema Std. 12389.

Callithama Wst. 3721.

Callithammion J. Ag. 580.

Callophyllos Beckin. 10443.

Callopil ophorum Don. 453.

Calobotra Std. 12056d.

Calochortus Pursh 3410.

Calocorthus Dumort. 3410.

Calophanos Wst. 8115.

Calorrhabdos Wst. 8023.

Calorrhexia Wst. 11635 b.

Calosteca Isis 2947.

Calostrophus Wst. 3282.

Calpigyne Blume 11068/1.

Calpurnea Std. 12413.

Caltha Burm. 5605a.

Calusparassus Jacq. \& H. 4667.

Calvaria Gärtn. f. 12658/1.

Calycoplectus Wst. 11556.

Calydermus Std. 7829.

Calygonium Dtr. 11682.

Calypogea Dumort. 2122.

Calyptrophora Wst. $9057 \mathrm{~b}$.

Cambderia Wst. 8838.

Camderia Dumort. 3665. 
Campanuloides H. kew. 6590.

Campesia W. \& A. 12291.

Campilostachys A. Juss. 76i)5.

Campylopteris Sternb. 2305.

Campylostemon E. Mey. 8222a.

Canacorus Endl., Wst. 4286.

Cantharulus Beckm. 1998.

Cantua Juss. 7791.

Caprosma D umort. 6642.

Carcia Räusch 10978.

Cardamane Wight 9515.

Cargyllia Std. 8576.

Carichtera Dumort. 9675.

Carlotea Arr. 12834/1.

Carludowica Wst. 4408.

Carmorea Std. 7642b.

Carpolepidium Lem. 2137.

Carpoliza Std. 3713.

Carpophyllum Std. 10037.

Carpophyllus Neck. 10037.

Cartamus Beckın. 6243.

Caruelia Parlat. 3460/1.

Caryococca Willd. 6807.

Casiostega Rupr. 2895/1.

Cassiera Räusch. 10498.

Castra Arrab. 6359.

Catanga Aubl., Std. 12836/1.

Catas Juss., Std. 5179. 5180.

Catasetum Dtr. 2864.

Cataterophora Std. 2771b.

Catestecum Dtr. 2864.

Cathastrum Turcz. 10770/1.

Catocarpaea DC. 9667a.

Cattleia Std. 3883.

Caudicia Hamilt. 7150.

Cavaria Puiz \& P. 12838/1.

Cellipora Linn. 630.

Cennarrhenes Std. 5159.

Cenorrhachis Wst. 5960c.

Centhriscus Spreng. 8970.

Centopodium Std. 4943.

Centosteca Isis 2787.

Centropodia R. Brown 2919d.

Cephalotrophis Blume 4718/1.

Cephaloxyes Spach 3345c.

Cephalozya Wst. 2135.

Ceramacarpus W'st. 9019.

Ceranthus Iinn. 2071.

Ceraselma Wst. 10877.

Cerasites Std. 9459.

Cerasoides Wst. 11968.

Cercostylus Wst. 5825.

Cervillea Sch. Bip. 5597.

Chaenanthera Dumort. 6302.

Chaerefolium All. 8972.

Chaetagastra Crüg. 11617.
Chaetocapnia Sweet 3496/1.

Chaetoceras Kütz. 116. 612.

Chaetoceros Ehrenb. 116.

Chaetopappus Sch. Bip. 5435.

Chaetotropsis Dtr. 2833.

Chamaea Griseb. 10592 d.

Chamaecnide Miq. 4777b.

Chamaecyparissias Wst. 5894a.

Chamaelaea Burm. 10917. 11270.

Chamaelancium Dumort. 11828.

Chamaela u c i um Desf. 11828.

Chamaemelanium Ging. 9782d.

Chamaenerion Gray 11496a.

Chamaeranthemum Nees 8238.

Chamelaeagnus Dod 4653.

Chammisoa Dtr. 4883.

Chantransia DC. 377.

Chapmania Wst. 12221.

Chaquepiria Std. 6944c.

Charidion Bong. 11276/1.

Chascolythrum Dtr. 2946.

Cheilanthoides Ung. 2293 (b).

Cheiradoplectrum iVst. 4168.

Cheirantus All. 9500.

Cheirianthus Dumort. 9500.

Cheirina Dumort. $9590 \mathrm{c}$.

Cheirostemum Std. 10241.

Chelona Dumort. 4832.

Chevaliera Beer 3793/1.

Chilogramma Wst. 2372.

Chinotria Dtr. 10537.

Chiocca Dtr. 6718.

Chisogeton Std. 10558.

Chlonanthes Dtr. 7555.

Chloraster Haw. 3742b.

Choerophyllum Brongn. 8972.

Choribena Std. 11352.

Choriozandra Std. 3135.

Chorisandra Wight 11093/1.

Chorosema Brongn. 12004.

Choteckia Std. 7441.

Chrascolytrum Isis 2946.

Christea Wst. 6198a.

Christiannia Wst. 9829.

Christima Std. 6866.

Christotelea Dtr. 9599.

Chromolepis Cass. $6254 \mathrm{~d}$.

Chrysanthos Fries 4793b.

Chrysolyga Wst. 11541b.

Chrysoraphanus Std. 9503b.

Chrysorhodium Std. 9768a .

Chytra Gärtn. f. 8039 .

Cienkowskia Reg. \& R. 10783/1.

Cinclidum Dumort. 2185.

Cistela Blume 3972.

Cistellaria Schott 12665/1. 
Cisthus Beckm. 9768.

Citrosena Bosc 4989.

Cladogynus Wst. 10959.

Cladostachis Std. 4878.

Cladostylus Wst. 7768.

Cladotrichum Std. 12456.

Cladrastris Std. 12417.

Clausenia Dumort. 10529.

Clavaria Std. 126058/1.

Clavi ceps Tul. 1694.

Clavula 1)umort. 3229.

Cleachne Rol. 2748.

Cleithria Std. 6185.

Clerodendron Burm. 7586.

Clethra Bert. 11414.

Clethria Fries 1675c.

Cletria P. Browne 1675c.

Clomopanus Std. 12584.

Clompanus Aubl. 12584.

Clonodia Griseb. 10629/1.

Closterost y les Torr.\& Gr. 11915c.

Clovesia Wst. 3947/2.

Clowesia Lindl. 3947/2.

Clypeola Burm. 12366.

Cnema Wst. 9305n.

Cobea Dumort. 7792.

Coc cocipsilum P. Browne 6929.

Codonachne W. \& A. 2370.

Codonia Wst. 10505a.

Codonocrinum Willd. 3436.

Coelor a ch is Brongn. 3043c.

Coelorhachis Endl. 3043c.

Coelorrhachis Wst. 3043c.

Coilotapalus P. Browne 4708.

Colchitropis Std. 12303a.

Colebrockia Std. 7442.

Colebrookea Smith 7442

Colebrokia Spreng. 7442.

Colletia Scop 4686d.

Collococcus P. Browne 12068/1.

Colobanthium Rchb. 2906a

Colobanthus Trin. 2906a.

Colocassia Wight 4354.

Colpophyllos 'Trew. 7797.

Columbea Std. 4547a.

Colus Räusch. 7431.

Colvilleia Std. 12454.

Comelina Bunge 3298.

Cominia P. Browne 12068/2

Comollia Wst. 11583.

Coniocarpum Wst. 950.

Coniosporum Wst. 1193.

Conobaea Bert. 4954.

Conogyne Wst. 5903.

Conomorphida Miq. 8508 a

Convolvolus Beckm. 7753.
Coppensia Dumort. 4007.

Cor Indum (Herm.) Burm 10641.

Cordia Rehb. $1010 \%$.

Coruucopieae Dumort. 2721.

Cornus Burm. 8530. 11739.

Corocephalus Dtr. 4711.

Corraea Sm.?, Re uss 11356.

Coryuites Wst. 12164.

Coryphanta Müll. 10017b.

Coryphantha Engelm. 10017b.

Cottaea Endl. 2861.

Cottonia Wight 4025/1.

Coublandea Std. 12406.

Coumarouma Wst. 12399a.

Coupia Dtr. 10797.

Courataria Dum ort. 11814.

Courtarea Itr. 6889.

Coutubea Std. 7380.

Crassina Scep. 5675a.

Creolybus Wst. 9888b.

Crepalia Schr., Std. 3003.

Criptocoryne Wight 4329.

Crisistrix Bosc 3136.

Crisium Lachen. 6257.

Crista pavonis Breyn, Burm.12442.

Crithamus Wst. 8798.

Crithesium Wst. 3018b.

Crossopterix Wst. 6897.

Cruckshankia Std. 6676.

Cruickshanksia Std. 11410.

Crypsocalyx A. Rich. 12048e.

Cryptodia Walp. 5600.

Cryptodon R. Brown 2178.

Cryptostoma Dtr. 8593.

Cujavus Rumph. 11783.

Cullenia Wight 10237/1.

Cumarouma Std. 12399a.

Cumbea Buch. 11812.

Cuscuta Linn. Cliff. 4872.

Cusparia Dtr. 12505.

Custenia Std. 10802.

Cuveraca Jon. 10586'b.

Cyanorchis Pet.-Th. 3285.

Cyathosphaera Dumort. 1702 (c).

Cybianthoides Miq. $8510 \mathrm{c}$.

Cyclium Std. 8031.

Cyclobothria Dtr. 3410 b.

Cyclopappus Cass. 5503/1.

Cyclopsis Wst. 6288a $\alpha$.

Cymballaria Std. 7917a.

Cynoxylum Pluk. 9096.

Cyrstostylis Dumort. 4206.

Cyrtandria I)tr. 8361.

Cyrtorrhyncha Wst. 9391.

Cystidium Iindl. 2526.

Cyticystus Burm. 9770a. 
Czernia Std. 2850.

Czernievia Std. 8886.

Dactylograma Link 2818.

Dactyloides Se r. 9186d.

Dactylorrhiza Std. 4077.

Dactylostegium Nees 8246/1.

Dactylotenium Dumort. 2868.

Dadia Arrab. 6250/1.

Dahlbergia Vahl 12381.

Dalechampsia W'ight 10884.

Dalzellia Wight 4647.

Damarites End l. 4561.

Dammarites Pres 14561.

T)angervillea Std. 11324.

Danthia Std. 11484a.

Dasianthera Pres 19830.

Dasyanthera Rchb. 9830.

Dasyanthus Wst. 8649 Bg.

Dasycarpus Örst. 10325/1.

Dasycoleum Turcz. 10570/1.

Dasyochloa Willd. 2923.

Dasyranthus Raf. 6073.

Dasytricha Wst. 400 .

Davallioides Ung. 2293 (a).

Day's Dumort. 5099.

Deastella Std. 5144b.

Decachaita Wst. 5343.

1)ecarrhaphe Wst. 11694.

Delia Dumort. 10109.

Demidoffia Wst. 7771.

Dendrostellera Meyer 5090b.

Deneckia Sch. Bip. 5563.

Denneckia Std. 5563.

Dentidea Std. 7430 .

Depretzia Dtr. 2744

Desfontaina Std. 11168.

Desforgia Std. 9235.

Desmaretia Du mort. 514 .

Desmazeria Dumort. 2929.

Diacidia Griseb. 10630/1.

Diadenia Wst. 345.

Diapedium Kön. 8246 .

Diapenzia Dumort. 7794.

Dibrachia Std. 11407k.

Dicerocaryon Dtr. 8269.

Dichoespermum Wight 3318/1.

Dichopus Blume 3834/1.

Dichospermum Müll. 3318/1.

Dicksonioides Ung. 2293 (c).

Diclidopteris Brack. 2380.

Diclyptera Dtr. 8246.

Dictyopeplus Wst. 1683.

Dictyospermum Wight 3299/1.

Didesmis Loud. 9687.

Didyplosandra Wight 8132 .

Dietrichia Räusch. 4257.
Dignathe Lindl. 3908/1.

Dilobus Std. 8307b.

Dimenostemma Std. 6503.

Dimeresia Juss. 10662.

Dimitopia Dtr. 8740.

Dimorpha Dtr. 9174.

Diperis Wight 4132.

Diphyllea Dtr. 9443.

Diphylus Std. 8649 I)g $\beta$.

1)iplandra Bert. 3587.

Diplarhena Agdh 3624

Diplasium Wst. 2445.

Diplatherum Brgn. $3065 b$.

I)iplocea Raf. 2923a.

Diplochaeta Wst. $3151 \mathrm{~g}$.

Diplochilus Std. 4106.

Diplochonia Std. 10047.

Diploclinium Lin d l. 9991.

Diplocoea Std. 2923a.

Diplodina Westend. 1798b.

Diplodiscus Turcz. 10341/1.

Diplolepium Dumort. 7281 .

Diplopteris Griseb. 10600.

Diplos a ster a Tausch 5716 .

Diplosastra Wst. 5716.

Diplosudon Dumort. 11555.

Diptanthera Schrank 8232.

Disanthera Beckm. 8232.

Dischoriste Dtr. 8114.

Disc o p odium Hochst. 7845.

Tiseris Wight 4132.

Disodia Dumort. 6737.

Disporium Leman 1454.

I)issorrhynchium Wst. 410 ?

Distachya Brgn. 3085a.

Distepha na Juss. 9872c.

Distephania Std. 9872c.

Disynapheia Std. 5339.

Ditrichum Wst. 2214.

Ditroche E. Neyer 10715.

Dodonea P. Browne 10699.

Döllia Sch. Bip. 5546/1.

Dolichonemia Std. 12460.

Dontospermum Sch. Bip. 5595.

Dontostenium Std. 9584.

Doranthera Std. 7953.

Doroceras Std. 8341.

Dortmannia Std. 6565a $\beta$.

Doupea Dtr. 9663.

Dracontopsis Lemr. 5703.

Draparnalia Ritg. 343.

Driandra Corr. 10992.

- Dumort. 5185.

Drogouetia Std. 4776.

Dryados Tausch 11943a.

Dudresneya W'st. 635. 
Duhaldia Sch. Bip. 5511.

Dulcichium Wst. 3233.

Dumortieria West. 1702.

Durieva Std. 7976.

Duvernoya Wst. 8202.

Dyanthus P. Browne 10141.

Dychotria Raf. 6692.

Dyplotaxis I)C. 9667.

Ebermiera Wight 8094.

Ecballium D u mort. 9930.

Echinoloma Std. 5239.

Echinopsis Linn. 6203.

Echinostachys Beer 3761/2.

Echyrospermun Schott 12683/1.

Edwarsia Dumort. 12408.

Eicosia Blume 4251/5.

Elachothamnus Wst. $5539 ?$.

Elaeachnus Burm. 5131.

Elaeagrus Pall. 5131.

Elaeococcos Wst. 10992.

Elaeococcus Std. 10992.

Elaiocarpos Burm. 10354.

Elaphomices 1)umort. 1599.

Elatostoma Wight 4747.

Eleanthus Wst. 3878.

Elemifera Linn. 12683/2.

Eleuthrantheron Std. 5682.

Eligia Dumort. 3230 .

Elshotzia Roxb. 7445c.

Elu th eria Linn. 11029.

Elythraria Dtr. 8091.

Elythrophorus Dum or t. 2965.

Elytregia Isis 3006.

Emelia Wight 6130.

Emerus B urm. 12158.

Encephalartus Std. 2654.

Endictya Ehrenb. 97.

Endivia (. Bauh. 6383.

Endogenites Brongn. 4508.

Spreng. 2594. 4510.

Enemium Std. $9419 \mathrm{c}$

Fnicostemma Std. 7379.

Epetorhiza Std. 7830c.

Ephialum Wst. 7593.

Epifegus Std. 8451.

Epilatoria Comm. 6520/2.

Epirrhizanthus W'st. 8466.

Epithecia Knowl. \& W. 4076/1.

Epitrachis Wst. 6258.

Equisitum P. Browne 2271.

Eranthus D nmort. 9417.

Eremiastrum Wst. 8643a.

Eremosyce Std. 9179.

Ericilla Std. 10180.

Eriocarpa I.ag. 5896.

Eriochaeta Fig. \& Not. 2771/2.
Eriochylus Std. 4209.

Eriocoila Wst. 7399.

Eriocoryna Std. 6212c.

Friogyne Std. 11951e.

Eriolithis Gärtn. 11969/1.

Erioloma Wst. 11574c.

Eriophytum Std. 7531 .

Friosphaera I)tr. 5896.

Eriosphaeria Wst. 605?.

Eriostemum Colla 10354. Std. 7515a.

Erithia Wst. 5568e.

Erithroxylum Juss. 10637.

Eriudaphos Wst. 9818.

Erochloa Std. 2932.

Erodendron W'st. 5143.

Erophoron Wst. $9186 \mathrm{f}$.

Ertelia Std. 11329.

Erythroropalum Blume 9960.

Erythrorrhiza Std. 8730.

Erythroxylum P. Browne 10637.

Escholtzia Du m o rt. 9468.

Espletia Std. 5 ti46.

Eteriscus Std. 6967.

Ettlingera Std. 4621 .

Eualisma Seub. 3330a.

Euatraphaxis Jaub. \& Sp. 4945a.

Eubanisteria Griseb. 10612 (b).

Eubrachyrhynchus Std. 6156a.

Eubrachyrrhynchus Wst. 6156a.

Euburdachia Griseb. 101323a.

Euburmannia Seub. 3604a.

Eucalypta D u un ort. 2225.

Eucamarea Griseb. 10592c.

Eucannavalia Std. 12303b.

Eucapnus W'st. 9479a.

Euchamaefistula End1. $12641 \mathrm{~b} \beta$.

Enchara Braun 461a.

Euconomorpha Miq. 8508c.

Fudiandra Dumort. 5015.

Endiarthron Mey. 5091.

Eudictya K ütz. 97 .

Eugesnera Hanst. 8417b.

Eugillia Wst. 7787c.

Euhediotis Std. 6813d.

kinheraclium Wst. 8921b.

Euheteropteris Griseb. 10608 (c).

Enlecythis Berg 11816a.

Eumaclura Blume 4696a.

Eu m a millaria Eng. 10017a.

Eumammillaria Müll. 10017a.

Eumascagnia Griseb. 10606/1c.

Funitella Brann 460a.

Luparaea Strl. 8499.

Eupecopteris Sternb. 2314a.

Euphocarpus Anders. 11356. 
Euphorbio - Tithymalus „Burm. 10877.

Euphorona Arrab. 12852/1.

Euphrobosces Griff. 4061.

Euproboscis Rehb. f. 4061.

Euria Ca mbess. 10392.

Eurudbeckia Walp. 5702a.

Eurynoma Std. 6732.

Eurystomum Wils. $2260 \mathrm{f} \alpha$.

Eurytium Wst. 631b.

Eustrephia Dtr. 3694.

Eutetrapteris Griseb. 10607 (a).

Eutriania Dtr. 2883.

Euvellosia Seub. 3676b.

Eveleyna Std. 3805.

Evonymadaphne Wst. 5024.

Excoecaria Linn. (lege) 10894.

Exoecaria Dumort. 10894.

Extinctorium Dumort. 2225.

Exytelma Dumort. 7257.

Fagaras Burm. 11304.

Fagelia Schwenck. 7904 .

Falconera Wight 10909.

Falkea Kön. 9976.

Falopia Wst. 12688.

Favrodina Reyn. 12852/2.

Feliciana Wst. 11806.

Fero lia Barr., Aubl. 11978a.

Ferrandia Gaudich. 9274.

Ferreiria Wst. 6886.

Festucaria Fabr. 2937a.

Ficinea Wst. 3216.

Figalonopsis Wst. 5556 .

Fimbrystylis Dtr. 3219:

Fischera Wst. 7291.

Flemingia Roxb. 7377. 12345.

Flessera Adans. 7498 .

F'os clitorius Burm. 12270 .

Forskolia Wight 4775 .

Foterghillia l)umort. 9128.

Fouguiera 1)tr. 9806.

Foxia Parlat. 3464/1.

Fracastora Adans. 7507.

Fraga Lapeyr. 11915.

Frankia Bert. 11102.

Freemamia Std. 6068 .

Freesea Std. 364?.

Frostia B ert. 2693.

Frumentum Matth. 4951.

Fuchsia Swartz 6799.

Fucoides Brongn. 817.

Fulchironia Lestib. 4482.

Funckia Du mort. 3418.

Galactea Wight 12291.

Galathea Salisb. 3617.

Galeopdolon Dumort. 7519b.
Galeottia Rupr. 311 .

Gallasia Mart. 11694 (b).

Galumpita Blume 4690/1.

Gandichaudia 1)umort. 10595.

Garaleum Walp. 5459.

Garapatica Karst. 6803/1.

Gardinia Bert. 3466.

Gassoloma I)tr. 5139.

Gastrodium Dumort. 4134.

Gastrorchis Blume 3395b.

Gavila Wst. 4226.

Gavillea Std. 4226.

Gaylussacea Wst. 8699 .

Geissorrhiza Dtr. 3653.

Geoffraea Linn. 12393.

Geoffroea J a cq. 12393.

Geoherpum Willd. 6746.

Geoides 'Tausch 11915b $\beta$.

Gerascanthos Std. 7637a.

Gerontopogon Gesn. 6409.

Gerostemum Std. 7:346b.

Gesnera P l u m. 8402.

Gesneria Linn. 8402. 8417.

Gesnouisia Std. 4767.

Glandonia Griseb. 10630/2.

Gledisia All. 12545.

Glochidinopsis Std. 11107.

Glochidium Wst. 11108.

Glossostemum Std. 10313.

Glychyrriza All. 12133.

Glypholecia Nyl. 1039.

Glyscosmis Dtr. 10522.

Gochnatea Std. 6288.

Goldenia Räusch. 7651.

Gomortegia Wst. 5019c.

Gomphraena J a cq. 4,921.

Gonianthes D) tr. 3605.

Goniolippia Schaner 7561b.

Gonobolus 1)tr. 7287.

Gonostemum Wst. 7346b.

Gontarella Gilib. 9419.

Gorostemon Std. 7346b.

Gossipium Herm. 10215.

Govana All. 10856.

Govindooia Wight 4156/1.

Gräffenrieda Dtr. 11717.

Grammalia Dumort. 723.

Granata malus Burm. 11820.

Graphophorum Dtr. 2849.

Grenwillea Std. 11407a.

Griselea Dtr. 11550.

Grönlandia Gay 4311/1.

Grumelia Dumort. 6706.

Grunilea P o ir. 6706.

Guajavos Burm. 11783.

Guapina Std. 7600 . 
Guaria Dumort. 10571.

Guilandia P. Brow ne 12441.

Guillandina All. 12441.

Guirea Std. 11450.

Guiterrezia Walp. 5809.

Guldeustädtia D um ort. 12183.

Guroa Hamilt. 7197.

Gurua Hamilt. 7197.

Gussonia Dtr. 9045.

Guttierrezia Wst. 5809.

Gymnactis Cass. 5775.

Gyınnima Wight 7313.

Gymuccalyx Schmidt $7469 d$.

Gymnoloma Bartl. 5708.

Gymnolomia Wst. 5709.

Gymnospermum Wst. 9445b.

Gymuostrix Dtr. 2771b.

Gymnosyphon Dtr. 3612.

Gynanthistrophe Std. 12467a.

Gyrenia Knowl. \& W. 3496/2.

Gyrostemum Std. 10185.

Habrantus Dumort. 3687.

Hachenbachia Dtr. 3658 .

Hackelia 0 p iz 7713.

Haemadiction Std. 7152.

Haematostrobos Wst. 2684.

Hänslera Std. 6382.

Halenea Wight 7410.

Halicacabum J. Bauh. 10641.

Halimolobos Tausch $9586 \mathrm{~h}$.

Halimolobus Wst. 9586h.

Halimus P. Browne 12695/1.

Haliserites Sternb. 831 .

Halmiltonia Dumort. 5036.

Halogeiton Wst. 4864.

Halyserites Un g. 831.

Hambergeria Wst. 11455.

Hapalaria Dumort. 1369.

Haplopteris Presl 2370.

Haquetia 1)tr. 8776.

Harpeloma Wst. 12053.

Hartigsea Std. 10560.

Hartigsia Wst. 10560.

Harwaya Std. 8051.

Hasselquitia Dumort. 8931.

Hateranthia Juss. 7899.

Havannella Linn. 12695/1.

Hebesinapis DC. $9661 \mathrm{~b}$.

Hecatounia Poir. 9406a.

Hedystachys Dumort. 8025 i.

Heinzelmania Neck. 7969a.

Helemonium Std. 5688b.

Heleogonus Wst. 3230b.

Heleophylax Std. 3229.

Helicmotrichum Std. 2908.

Helicotrichum Bess, 2908.
Helicterioides Std. 9895a.

Heliopliylax Std. 3229.

Helitophyllum Wst. 5177.

Helixira std. 2623.

Helmintochortos Link 730.

Heloscia Dumort. 8791.

Hemicarpus F. Müll. 8743b.

Hemidemus Dumort. 1798.

Hemilobus Wst. 8307a .

Hemistephia Std. 6212b.

Hemitalia Dumort. 2532.

Henselera Rchb. 6382.

Heriteria Dumort. 3665.

Hermodactylum B a ${ }^{\circ} \mathrm{l}$. 3398b.

Hernandezia Hfmsg. 5119.

Herpestes Link 7978.

Herpestis G ärtn. f. 7978.

Herpetium Wst. 9782 f.

Herpetospermum Std. 9964.

Hertella Std. 5119.

Heteracea Std. 6466.

Heteranthesis Wst. 7496b.

Heterelytrum Wst. 3072a $\alpha$.

Heterocarpus Wight 3299/2.

Heterochilum R ch b. f. 4014c.

Heteroptera Marg. \& R. 9042/1.

Heterostemum Std. 12476.

Heterozyges Wst. 11385.

Hexabolus Std. 9326.

Hexopea Std. 3861.

Hexorina Std. 3387.

Hibanthus Dtr. 9783a.

Higgensia Std. 6927.

Hil dbrandtia Nardo 623.

Hildenbrandtia R ch b. 623 .

Himanthophyllum 1)tr. 3704.

Himenanthus Std. 7846.

Hinghstonia Std. 5753.

Hiusia Wst. 6850.

Hirnelia Wst. 5998.

Hirtellia I)umort. 11971.

Holmskidia Inmort. 7594 .

Holocheilos Wst. 6359a.

Holopetalon Gris.(10612d)10608(a).

Homalanthes Std. 10907.

Homalanthus Wst. 5956.

Homalia Wst. 2265e.

Homoeantherum Std. 3093.

Homoioceltis Blume 4687/1.

Homomelis Wst. 9125.

Honckeneya Std. 10335 .

Hoorebeckia Std. 5500a. •

Horustedia Juss. 4261.

Hosakia Grah. 12053.

Hugelroea Std. 12013.

Humboldia Rehb. 12480. 
Huszia Klotzsch 9973.

Hutchintia Bory 723.

Hutschinia Dtr. 7340 b.

Hybiscus 1)umort. 10205.

Hydrastylis Std. 3620.

Hydrocarpus Dtr. 9847.

Hydrolaea Dumort. $78(17$.

Hydrophaca Hall. 4322.

Hydropityum Std. 7974.

Hydrotrida Willd. 7978.

Hygromitra Fries 1897a.

Hy manthalia Dumort. 5:?

Hymenanthus Dtr. 871:3e.

Hymen a therum Cass. 5791.

Hymenolobium lienth. 1:8966/1.

Hynemophyton Dumort. 2006.

Hyınenospermum Bentli. 8082/1.

Hymenostomia W'st. 2513.

Hypelythrum Dtr. 3200.

Hypericoides P lum. 10458.

Hypuaca Bory 681 .

Hypobrychia W'st. 11535.

Hypochnaena Wst. 1591.

Hypopogon 'I'urez. 10412/1.

Jabrosa Std. 7848.

Jacobaca Burm. 5573.

Jaltonia Std. 7828.

Jambos Burm. 11773.

Jamesia Nees 6432.

Jan-Raia Plum. 3581.

Janraja Ad ans. 3581.

Jaraphaea Std. 11573.

Jasninanthes Blume 7:314.

Jasminum Burm. 6711.

Ibbertsonia Std. $11992 \mathrm{~b}$.

Ichtlyothera Wst. 5632.

Ichtyosma Std. 2675.

Icoturns Wst. 11951a.

Ifdregea std. 8903.

Ilaea Dumort. 41:.

Illa Adans. 7552.

Inophyllum $\mathrm{B} \mathrm{urm.} 10413$.

Int ybus C. Bauh. 6383.

Joosia Karst. 6876/1.

Josephia Wight 4061/1.

Ipomea A 11. 7751.

Ireum Burm. 9780.

Irillium Rafin. 3538.

Isanthus DC: $636 \% \mathrm{~b} \eta$.

Isartia Dumort. 6804.

Isias Not. $4126 \mathrm{~b}$.

Isostoma Dtr. 6572.

Isquierda Willd. 8530 .

Isquierdia Poir. 8530.

Jujuba Burm. 10821.

Julostylis Thwait. 10217/1.
Juno Tratt. 3626 .

Jurinaea Std. 6271.

Ixalum Forst. '2782.

Izquierdia Ruiz \& P. 8530.

Kagenackia Std. 11958.

Kallymena Wst. 658.

Karamyschovia Std. 6825.

Karthemia Walp. 5572.

Kaulfussia 1)enust. 10745.

Kennedynella Std. 1228\%.

Kentia Blume 4433. 9324b.

Kerandrenia Std. 10279.

Kicksia W'st. 7917b.

Kirganelia l)umort. 11099.

Kitaibella Reuss 10190.

Kinifa Adans. 10458. 10459.

Koulda-Pail P'lum. 4325.

Kokera Adans. 4883.

Kordellestris W'st. 8307.

Kreyssigia Wst. 3:35.

Kundanmamia std. 8838 .

Kuntia I)umort. 4424.

Labourdomaea Wst. 85.76.

Laccopteris G ö p p. 2545 .

Stermb. 2544.

Lachryma Jobi Burm. 2717.

Lacuris Std. 11304b.

I adanum Tausch 9768.

Laenecia Walp. 5533.

Laetji Osb. 10673.

I amiarckea Std. 7862 .

Lamourouxella Bory 353 .

Lampocarpya Spreng. 3194.

Lamprococcus Beer 3760/1.

Lamproconus Lemr. 3772d.

Langefeldia Std. 4747.

Larbraea Dumort. 10137.

Lasiorrhegma Wst. 12461g.

Lasiotrichos Lehm. 2732.

Laudtia Wst. 6189.

Laugeria Li in n. $6753 \mathrm{~b}$.

Lauroceras us Tourn. $11967 \mathrm{c}$.

Laurophillus Röm. \& Sch. 11201.

I.eachea std. 5721.

Jeawenworthia Dtr. 9519.

Lebitinia Std. 5787.

Leciscium Gärtn. f. 12705/1.

Lecostomum Std. 11982.

Jedon Tausch 977\%).

Leeuwenhockia Std. 6662.

Lehmamia Jacq. 7120.

$$
\text { - Tratt. } 11915 .
$$

Leiachnaea Std. 6088b.

Leicesteria Wst. 6985.

Leiophyllum Wst. 4612e.

Leochilus $\mathrm{Know}$ l. \& W, 4008. 
Leonura Ust. 7481.

Leoptoda I)umort. 5807.

Lepachis Std. 5704.

Lepargyraea Std. 5129.

Lepechina Dumort. 7476 .

Lepeostegeres Endl. 9114p.

Leperrhiza Wst. 3717.

Lepichosma Suith 2414c.

Lepicolea 1)umort. 2114 b.

Lepicystis Smith 2460b.

Lepidolaena Dumort. 2113.

Lepidota Sternb. 2633 (a).

Lepitoma Torr 2941.

Lepostegeres Blume $9114 \mathrm{~b}$.

J.eptalium Sweet 9598.

Leptaninium Std. 8451.

Ieptaspis R. Brown 2711.

Leptocnide Blume 4752/1.

Leptomon Std. 11024.

Leptonychia Turcz. 10316/1.

Leptorrhabdus Wst. 8038.

Leptorrhachis Wst. 10923.

Leptorrhynchus Wst. 6044.

Leptosura Blume 4696b.

Leptotaen ia Nutt. 8902.

Leptotherium Dtr. 3050.

Leptothrium Kunth 3050. 3878.

Leptotrina Wst. 10068.

Leptranthus Std. $6236 \mathrm{~h}$.

Lettosomia Dumort. 10397.

Leucacantha Wst. 6236y.

Leucandron Std. 5143.

Leucanotis Dtr. 7074.

Leucocarpum A. Rich. 9815.

Leucorhodium Std. 9768a.

Levisia Std. 10084.

Lichinora Wight 3852.

Lidınodorum Dumort. 4228.

Ligfootia Dumort. 6590.

Ligustrum Burm. 7082.

Lilacum Ren. 7009.

Limbaria Wst. 920.

Limnostachys F. Müll. 3402/1.

Limo Rumph. 10535.

Limonellus Rumph. 10535.

Linanthe Dumort. 8607.

Lindernia D. Ion 7989.

Linophyllum Pont. 5069.

Linopteris Stermb. 2291.

Linospartum Std. 2714.

Jiperanthus Dumort. 4214.

Lithocarpos 'Targ. 4491.

Litria Hook. 11198.

Litsaea Nees 5052 .

Littlea Dumort. 3751b.

Lobostemurn Wst. 7662.
Lomathophyllum Dumort. 3435 .

Lomenia Pourr. 3656/3.

Lomoria Dumort. 2361.

Longchampsia Wst. $6106 \mathrm{~b}$.

Lopanthus Vitm. 10296.

Lophatherium Wst. 2964.

Lophozonia Turez. 10646/1.

Lorentia Sweet 5311 .

Louichia Std. 10096.

Luchia Std. 3603/1.

Lupinoides Tausch 11915b/3.

Luxenburgia Std. 11272.

Lychnanthos Gmel. 10160.

Lychnanthus G mel. 10160.

Lycioserissa Röm. \& Sch. 6726.

Lycorus Loud. 3701.

Lygnia Dumort. 3273.

Lygurus Dtr. 2822.

Lyperanthus R. Br. (lege) 4214.

Lysimachia Burm. 7354.

Lysionothùs Dtr. 8336.

Lyssanthe Dtr. 8607.

Lytrostylis Wst. 7644.

Macclellandia Wight 11563/1.

Nachanaea Std. 10450.

Machenziea Nees 8182 .

Machura Std. 4696.

Mackaya Arn. 9960.

Macqueria Kunth 11304e.

Nacrocalyx Trew 7797.

Macrochordium Beer 3761/1.

Macroplodia Westend. 1798a.

Macrospermum Std. 9638.

Macrostemma Sweet 11501.

Macrostomia Wst. 7665.

Macrotonica Std. 7665.

Madhuca Gmel. 8552.

Magydoris Dtr. 9004.

Majanthemophyllum Vis. \& Mass. $3562 / 1$.

Majanthemum Siegesb. 3545.

Malacoxylon Jacq. 12711/2.

Malaspinea Wst. 8540.

Nalicope Vitm. 12711/1.

Malpigia P. Browne 10618.

Malteburnia Std. 2699.

Malvinda Burm. 10296.

Mamei P lu m. 10435.

Mammaria Ces. 1304.

Nianduyta Std. 11283.

Mangle Adaus. 11464a.

Mangles Plum. 11464a.

Mansana Gmel. 10821.

Napourea Wst. 6696.

Marasmium Wst. 1995.

Marcantia P. Browne 2085. 
Narcielia Std. 7732.

Margyriocarpus Dtr. 11933.

Marliera Dtr. 11749.

Marodenia Dumort. 7307.

Marshallia Gmel. :850.

Marsilaea D u mo rt. 2612.

Nartiniera Std. 10408.

Mascagna Std. 10606/1.

Mascagnia J3ert. 10606/1.

Maulia Wst. 3419.

Naundia F. Müll. 3327/1.

Mauricon Wst. 12309b.

Mazentoxeron Wst. 11356.

Meadea Std. 8486.

Mebora Std. 11148.

Mediastina Dod. 2039/1.

Megalurus Wst. 2967a.

Megaphyton Art., Endl. 2636.

Megaphytum Art., Sternb. 2636.

Meisteria Gmel. 10487/1.

Melanocarya 'Turcz. 10767/1.

Melanodendrum Std. 5415.

Nelanopsidium Colla 695 t.

Nelanoselium Dumort. 8952.

II elanospermum Dtr. 8952.

Melanthus Weig. 12719/1.

Meleagrinex Arr. 12875/1.

Melinus Dtr. 2766.

Nelitoxylon Hart. 4545/1.

Melonanthus Nüll. 7390d.

Nenesteria Dtr. 6939.

Menilis Dumort. 2766.

Menioccus Std. 9527.

Nenzelia Linn. 9888.

Mephrodium Dumort. 2491.

Merichea Wst. 11809b.

Nerista Banks 8515.

Meristastigma Std. 3640.

Neristostigma Dtr. 3640.

Merostachis Std. 2992.

Mesocentrum Std. 6236 Dy.

Nesosphaerium Std. 74370 .

Nesosphaerum P. B rown e 7437o.

Mesotetra Wst. 5838b.

Mespylus All. 11904.

Netachilon Std. 3858.

Nichauxia Räusch. 12875/1.

Micrauchenia DC. 6475a.

Microcarpea Dtr. 8009.

Micrococcus Beckm. 10343aß.

MIicrococos Burm. 10343a $\beta$.

Vicromelon Std. 10530.

Microrhyncus Wight 6449.

Microrrhachis Wst. 5960a.

Microrrhynchus Wst. 6449.

Microsorus Wst. 2469.
Microthea Juss. 10173.

Nielighoferia Wst. 2197.

Mirobalanus Std. 11440b.

Mischocaryum Wst. 5150a.

Mischolobium Wst. 12384.

Miscodendrum Std. 9103.

Viitostigma Blume 4133/1.

Mitrasachme Wight 7042.

Nitrocarpa Torr. 3230.

Mitrosphaera Dumort. 1702(t).

Nitrosporium Lev. 1294.

Mitrularia Dumort. 1915.

Mnniopis Dumort. 2143.

Mocquinia Std. 9114f.

MIönchia Wender. 2761.

Moghamia Std. 12345c.

Mokus in Cibot 1678.

Moldenhaueria Std. 4780).

Noldenhaweria Std. 12460.

Molkia Dumort. 7688 .

Noloppospermum Dumort. 8977.

Molospermum Std. 8977.

Mombin D C. 11223.

Momisiopsis Blume 4686e.

Monacanthus Sweet 3946b.

Nonacenta Std. 11600.

Monachme Dumort. 2761.

Monandraira Desv. 2906/1.

Monbin Plum. 11223.

Moniera P. Brow ne 7978.

Monneria Std. 7978.

Monniera Pers. 7978.

Monocistis Std. 4274.

Nonophylleia Wst. 8345.

Moringia Dtr. 12544.

Morisona Plum. 9733.

Morisonia Linn. 9733.

Normoraphis Jack 9044.

Morophorus Std. 4694.

Mosophylla Dumort. 2140.

Mucrenea Std. 4931.

Nuhlembergia Dumort. 2818.

Munrozia std. 6513.

Murex Linn. 8270.

Mursupella Dumort. 2127.

Mussaenda B u rm. 6914.

Mylachne Std 8451.

IIyosanthus Dumort. 10139/1.

Myrioneurum Std. 6978.

Myzodendrum Forst. 9103.

Naenratospermum Std. 4798.

Nagas Gis. 4300.

Naghas Mirb. 10442.

Nardurus R ch b. 3008.

Narvelia Link 9390.

Nasturium Dumort. 9503. 
Nauemburgia Std. 5781.

Nauia Borkh. 2239b.

Navenburgia Juss. 5781.

Nectaroscilla Parlat. 3457/1.

Nematopus Seub. 3295d.

Nemoursia Mér. 2081.

Neocies Std. 6126a.

Nephelephyllum Blume 3820.

Nerija Std. 10789.

Nessea Std. 11541.

Nestlera Willd. 2883.

Neudorffia Std. 7814.

Neuroloma Std. 2945.

Nevreda I)tr. 11963.

Nevropteris Brgn. 2286.

Nictanthes All. 7000 .

Nierenbergia Std. 7880.

Nilsonia Brongn. 2664b.

Nimbo Dennst. 10526.

Nimmonia Std. 9182.

Noccea Dumort. 5293.

Nodolara Imper. 632.

Noisettea Dumort. 9785.

Noranthea I)umort. 10455.

Noryska Std. 104591.

Nothoceras Dtr. 9497.

Notocheta Std. 7529.

Notopteris Griseb. 10606/1a. Presl 2312a.

Nowodworskia Std. 2833.

Nycterisitium Std. 8541.

Nycteristion Wst. 8541.

Ochrosma Dumort. 10240.

Ochroxylon Std. 11304d.

Octostemum Std. 11680b.

Octostomon Std. 11680b.

Ocymophyllum Buxb. 127:32/1.

Odontagnanthia Std. 6242b.

Odontostemum Std. 9532c.

Odostemum Std. 9449b.

$0 \mathrm{~g}$ c e r ostylus Cass. 5994.

Oliganthus Wst. 5252f.

Oligophyllum Wst. $6359 \mathrm{~b} \alpha$.

Oligostemon T'urcz. 11288/1.

Oliviera I)tr. 8796 .

Olythia Std. 11760.

Omphalocarpus S preng. 8563 .

Oncorrhiza Std. 3582.

Onobrychis Burm. 12239.

Onosmidium Dumort. 7686.

Opalatoa Isam. $12 ; 22$.

Ophiothrix Näg. 423.

Ophiur os Gärtn. f. 3034.

Ophiurus R. Brow n 3034.

Ophyurus Dtr. 3034.

Opodia Wst. 9018.
Oppoponax Dumort. 8916.

Opuntia Nacc. 439.

Oraoma 'Turez. 10556/1.

Orbiguia Bert. 11171/1.

Orcya Arr. 6524/1.

(Oreanthus Walp. I)eleatur!)

Orectospermum Schott 12732/1.

Origophragmus Dtr. 9665.

Orleana Herm. 9811.

Ormacarpum Wight 12211.

Ornithogaloideum Dtr. 3418.

Ornithorynchium Röhl. 9.576.

Orobanchia Vand. 8356.

Orobus Burm. 12346.

Oroxylon Std. 8280.

Orthopetalum Beer 3772c.

Orthophytum Beer 3772c.

Orthopleuria Sternb. 2314b.

Orthopogon A. Spreng. 2768b.

Orthosanthus Std. 3617.

Orthostemum Std. 7375.

Osbeekia Wight 11609.

Usteiza Std. 6525.

Othanthera Std. 11605.

Oudeneya Itr. 9502.

Ouragoga Linn. 6680.

Ourèt Adans 4901.

Ourouparea Wst. 6893e.

Outarda Dumort. 6889.

Ouviandra Dumort. 3329.

Oxybaphos Wst. 4968.

Oxyglycus Bowd. 12883/1.

Ox yrampis A. Juss. 12132.

Oxysepala Wight 3835/1.

Oxytropus Gray 12187.

Pachydendron D u mort. $343 \mathrm{fg}$.

Pachyrrhynchus Wst. 6086.

Pachyrynchus Std. 6086.

Paivaea Berg 11803/1.

Palna Plum. 4494.

Paltonophora Wst. 59.

Pancaseolus Caesalp. 8808

Pandanophyllum Walp. 4407/2.

Pankea Örst. 11524.

Panopis Std. 5164.

Panzeria Gmel. 7859.

Papaja Rumph. 9904.

Paptisia Dtr. 119.1.

Paractacmum Dumort. 2761.

Pararhysis Endl. 6229d.

Pararrhysis Wst. 6229d.

Parastreblus Blume 4719b.

Paratrophis Blume 4719/1.

Parkensonia P. Browne 12438.

Pasithoë Decne 863/1.

Pastorea Tod 9620/1. 
Patellaria Gray 1905.

Patomia Std. 9319.

Pattersonia Wst. 3638.

Pattonia Wight 3865/1.

Pavonia Domb. 7637.

Pechea Arr., Std. 8515. - Pourr. 2722.

Pectinea Gärtn. 12741/1.

Pectoraria Nees 8222b.

Pegamaea Duniort. 7034.

Pegamea Vitm. 7034.

Pegia C o lebr. 11192.

Peireskia Std. 10037.

Pela Rheede 11783.

Peliostomuin Ben th. 7951.

Pellinia Molin. 10381.

Peltaria Burm. 122266

Peltostegia Turcz. 10281/1.

Pemphidia Dumort. 808/4.

Pentapteris Griseb. 10607 (b).

Pentapterophyllum Wst. 11514a.

Pentastemum Std. 7933.

Pentestemon Dumort. 7933.

Penthanthus Std. 6349.

Pentorobum Beckm. 9175.

Pepronia Dumort. 4599.

Pereiria Wst. 9248.

Perepusa Std. 7394.

Peribea Dumort. 4724.

Perimenium Std. 5746.

Peripea Std. 8028.

Peristylis Wight 4093.

Perizoma Miers $7841 \mathrm{~b}$.

Permna Dumort. 7580.

Perostema.Räusch. 5027b.

Petasodes Spreng. 9395d.

Petellaria Dumort. 1905.

Peterospermum Wight 10315.

Petraea Ung. 11443.

Petrocalis Dumort. 9540.

Petrotlieca Rudge 12887/1.

Peurousea Std. 5949.

Phacellaria Willd. 2870.

Phaelypaea P. Browne 8452.

Phaenix Dumort. 4482.

Phaeocarpos Std. 10704.

Phaethusa Gärtn. 5753a.

Pliaetusa S ch reb. 5753a.

Phagnalion Wst. 5536.

Phaios Wst. 3895.

Phajas Wight 3895.

Phaloura Std. 2961a.

Pheboanthe Wst. 7547b.

Phegonium Ung. 4675.

Philippodendrum Poit. 10320.

Phillanthus All. 11093.
Phillipaea Wight 8452.

Phillis All. 6641.

Philodencirum Std. 4365.

Philodyce Std. 3294.

Philostemon Raf. 11199c.

Philostemum Std. 11199c.

Phipsia Wst. 2824.

Phiteuma All. 6604.

Phitolacca All. 10179.

Phlaeospora Westend. 1829.

Phlebolithris Std. 855.5.

Phlomostachys Beer 3772l.

Phragmitis Beck. 2850.

Phreatea Wight 38.51.

Phthecrospermum Std. 8060 .

Phylesia Dumort. 356::.

Pliyllactis Std. 5674.

Phyllites Brgn, 4320. Sternb. 4540.

Phyllobryum W'st. 4599 a.

Phyllodiastrum Walp. 12056b.

Phyllostachys Torr. 3122.

Physalobium Std. 12280.

Physanthera Bert. 3998.

Physarus Lindl. 4249/2.

Physema Rehb. 9880.

Physicarpus Wst. 12033.

Physingia Std. 3905.

Physocarpus Wst. 9392b.

Physocodon Turcz. 10303/1.

Physophora Hfmsg. \& L. 9017.

Physydrum Rafin. 44?.

Phytarrhiza Vis. 3777/1.

Phytocrena Std. 9293.

Picardenia Std. 5837.

Picrophleus Dumort. 7047b.

Picrorrhiza Wst. 8020.

Pieraea Walp. 5580.

Pierardea Wight 11088.

Pilobolus Braun 790/1.

Pinckeneya Dumort. 6865.

Pinckneya Rich. 6865.

Pinus Brgn. 4554 B.

Piperodendron Heist. 12747/1.

Piptochlaena Wst. 7657c.

Piptosaccos Turez. 10586/1.

Piptostegia $\mathrm{H}$ of fm s g. 7751.

Piptostemum Std. 63+3b.

Pistolochia Bernh. 9482.

Pistorina Dumort. 9162.

Pistorinoides Dtr. 9165b.

Pithecellobium Wst. 12578.

Pithecollobium Std. 12578.

Pithuranthos DC. 8839b.

Pityranthe Thwait. 10341/2.

Pityrophyllum Beer 3766/1. 
Pixidaria Isis $12755 / 1$.

Plagiocaryum Willd. 12857/2.

Plagiocheilos Wst. 5987.

Plagiopodia Dtr. 5169 Aa.

Platyclados Wst. 4519.

Platystachys C. Koch 3788.

Platystemuin Std. 9473.

Plazeria Std. : :160.

Plectocarpuin Wst. 1062.

Pleomeles Salisb. 3432.

Pleon anthus Ehrh. 1014la a.

Pleothea Std. 6979.

Pleragina Arrud. 12858/1.

Pleuroblepharon Std. 4250.

Pleurophus Dtr. 2592.

Pleuropteris Griseb. 10606/1 b.

Pleuroschisma Dumort. 2119.

Pleurostemum W'st. 11489d.

Pleurozia Duınort. 2116.

Plexaura Wst. 4169.

Plocaglottis Str. 3903.

Plotia Schreb. 2929.

Pluckenetia Std. 10927.

Podanthera Wight 4239/2.

Podisonia Dumort. 4306.

Podoriocarpos Lam., Std. 9726.

Podoriocarpus Wst. 9726.

Poecadenia Wst. 12125.

Poederia Reuss 6735.

Polyanthes Linn. 3754.

Polycandia Std. 10769.

Polycyrtas Wst. 8898a.

Polyphyllum Wst. $6359 \mathrm{~b}_{i}$.

Polypremnus Beckm. 8011.

Polyrrhaphis Wst. 2359b.

Polystachia Brgn. 3085b.

Pontederas Hfinsg. 3400.

Pontesia Arrab. 5312/1.

Poraqueiba Aubl 10487/1.

Porcella Dtr. 9:322.

Porodothium Wst. 963.

Possura Aubl. 9450/1.

Potamica Poir. 5160.

Pothos Adans. 3754.

Pothuava Gaudich. 3762.

Prescottia $\mathrm{L}_{\mathrm{i}}$ in d 1.4155.

Priaranthus Dumort. 7344.

Prinodia Griseb. 10808/1.

Protolepis Std. 5252b.

P s a mmoseris Boiss. 6456 .

Psammotrophia Meisn. 10075.

Pseudanthus Wight 4901/1.

Pseudoathrixia Wst. 6104b.

Pseudobasilicum Std. 12752.

Pseudocormus Wst. 1564c.

Pseudoelephantopus Std. 5286e.
Pseudostoechas Std. 748.9c.

Psilochaena Walp. 6.458d.

Psiodia Walp. 5480.

Psychechilus Wst. 4102.

Psychosperma Dumort. 4439.

Psydax Std. 6729c.

Psyl o xylon Ner. 11566.

Ptelia 1)tr. 11315.

Pterigostachyum Nees 3054.

Pterisma Presl 10740c.

Pterobryum C. M ï 11. 2260h.

Pterocarpus Berg. 12364.

Pterocladis Lamb. 5542.

Pterogenium Angstr. 225.c.

Pterophytum Wst.5725e.

Pteropodium Willd. 2\$39.

Ptichotis Dumort. 8790.

Ptileris Rafin. 6126a.

Ptilinium Wst. 8793

Ptilostemum Std. 6260a.

Ptilothus Dumort. 48.97.

Ptycheteropteris Griseb. 10608 (b).

Ptychophytlum A. Braun 2761.

Pulchia Arrab. 12892/1.

Purchia Dumort. 7586 .

Pycnocomon Wst. 6256.

Pycnocyclus Wst. 8993.

Pycnosandra Blume 11069/1.

Pyrotheca Std. 3665.

Pyxidaria Schott 1275.)/1.

Quesnelia Gaudich. 3793/2.

Raciocarpon Wst. 20) 30 .

Racosperma Wst. 12566 a.

Racotheca Wst. 2076.

Ragadiolus Beckm. 6371 .

Rangium Juss. 7007.

Raphelingia Dumort. 12302/2.

Raphioacme Wst. 7204.

Rauroifia Plu m. 7091.

Rauwolfia Linn. 70?l.

Razumovskia Wst. 910..

Reaumurea Std. 10471.

Reichardia Roth 6425. 7922.

Reiffenscheidia Std. 9361.

Reinera Dennst. 9085a.

Remigia Wst. 6874.

Renalmia Plum. 3777.

Renardia Turcz. 11174/1.

Renealmia Linn. (1737) 3777.

Rhabdospora Westend. 1829b.

Rhagocarpum Wiglit 75.9a.

Rhantherium Sch. Bip. 5566.

Rhaphedospera Wight 8250 .

Rhaphidosperma Don 8250 .

Rhaphiospora Theob. 1012.

Phaphistemma Std. 7231. 
Rhinanthus D. Don 8073. Rhinchothecum Dtr. 8363. Phipidium Wst. 437. 3066. Rhizocarpum Wst. 1006. Phizoctonum Leman 1458. Rhombol y trum Link 3097/1. Rhophostemoñ Wst. 4154. Rhyacophila Wst. 3505. Rhynchotoechum Blume 8363. Rhyssopterys Wst. 10613. Rhytidocarpaea Wst. 5938f. Rhytidosis Wst. 6038.

Ribeirea Arrud. 12892/3. Richnophora Leman 1958. Ricinocarpos March. 11001. Ricinokarpos Burm. 11029. Riedlia Dumort. 10298. Rincourtia Std. 5629 . Rizophora Burm. 11464. Robertsia Wst. 8542. Robinson ia DC. 6168 . Roccelia Dumort. 1091. Rodrigueza Dumort. 3997. Roëlloides Banks 6601 . Röperia Std. 11389. Rondoletia Dumort. 6844. Rophostemon Endl. 4154. Roptrostemon Blume 4154. Roscia Dtr. 11312.

Rosenbergia Urst. 7792/1. Rottbölia Dumort. 3043. Roubieua Carey 4806.

Roylea Nees 2925.

Rubentia Boj. 11310. Rulingea Dumort. 10282. Rumastra Wst. 4944c. Rupala Vahl 5175. Russegera Std. 8158. Russellia Wst. 7938. Ryana Wst. 9861. Rytidotrochis Blume 11558e. Rytiglossa Std. 8214. Sabbata Arrab. 6525/1. Saccophorum Wst. 2184. Saguaster major Rumph. 4449. Salmea Wst. 3432 .

Salpingea Dtr. 11662.

Samaria Wight 8530.

Samp a c ca Rumph. 9350. Sanguinella Gleich. 3)35.

Sanseveria Dumort. 3432.

Sarachra Dumort. 7828.

Saranthe Reg. \& K. 4279b.

Sarcobolus Dtr. 7316.

Sarcocnide Blume 4763g.
Sarcocordylis Wall. 2680. Sarcodactil is Gärtn. 10535. Sarcodactylis Std. 10535. Sarcopetalum Zoll. 9312. Sarcotheca Turcz. 11239/1. Sarna Karst. 2687/1.

Sarodonia Std. 7930b.

Sarracinia Wst. 9761.

Sarraziniา Hfmsg. 9761.

Sassa Bruce. Gmel. 12566.

Sassia Molin. 11420.

Saul Roxb. 1036?.

Saurauia Wst. 10398

Saxe-Gothaea Lindl. 4553.

Saxifragia Herm. 8812a.

Scabiosa Burm. 5252i.

Scalpellorchis Blume 4040 (b).

Scalpiopsis Std. 6052.

Scamone Dnmort. 7216.

Scepsothamnus Std. 6963.

Sceptranthes Std. 3683.

Schaenocaulon Benth. 3368.

Schaffnera Sch. Bip. 6320a.

Scherardia M u r r. 6637.

Schismocerus Std. 3855.

Schisolaena Pet.-Th. 10379.

Schistochila Dumort. 2139.

Schistotega Dumort. 2159.

Schizopteris Griseb. 10607 (d).

Schizotheca Ehr. 4315/1.

Schlerochloa Parlat. 2968.

Schlimia Planch. 4071.

Schlosseria Mill. 4954.

Schmidelea Wight 10649.

Schmidia Wight 8088/1.

Schnizleinia Std. 3678. 8856.

Schoberia Me ye r 4851.

Schönfeldia Wst. 2866.

Schoenobiblos Endl. 5089 .

Schoenobiblus Mart. 5089.

Schoenodoros Wst. 2967b.

Schradera Willd. 11029.

Schranckia Scop. Gmel. 10797.

Schweickerta Gmel. 7415.

Schweiggeria Wst. 10425.

Schweyckerta Gmel. 7415.

Schweyckherta Gmel. 7415.

Schyzophyllum I)umort. 1987.

Sciurus Dtr. 11324.

Scleromitrum Wst. 6814.

Scleropoa Dumort., Gris. 2970.

Scleropyrum Wight 5063.

Scleroxylon Std. 8515 .

Scobedia Iab., Std. 12769/1.

Scorias Wst. 11175.

Scottia R. Brown 12040. 
Scutea Wight 10828.

Scyphyphora Dtr. 6646 .

Sczegleewia T'urcz. 10270/1.

Sczukinia Wst. 7408.

Seafforthia Std. 4439.

Securigena Dumort. 11125.

Sedum Adans. 9171.

Seguaster major A uct. 4449. minor A uct. 4442.

Selenostylis Blume 4040 (a).

Semeionitis Std. 12:83.

Serialidia Fee 958b.

Sericophysun Std. 6064f.

Serigrostis Std. 2818.

Serjana Arrab. 12897.

Sertolara Nardo 439.

Serturnera Auct. 4921a.

Sherhardia Gled. 6637.

Shutera Wight 12290.

Shuttelworthia Std. $7558 \mathrm{~b}$.

Siegesbekia A 11. 5610 .

Sigisbeckia Dumort. 5610 .

Sigmatophylluin Dtr. 10614.

Silex Dumort. 8939.

Siphocampylos Wst. 6568.

Siphosphaera Dumort. 1702g.

Sissolia Rupp. 12197d.

Sisymbryum Dumort. 9586.

Sisynrichium (Herm.) Burm. 3626.

Sisyrinchium R. Brown 3619.

Skirrhoglyphis Wst. 5671b.

Skolemora Arrud. 12899/1.

Skytophyllum Wst. 2164.

Soberbaea Dtr. 3469.

Sobrus Reich. 11893f.

Solenanthe Wst. 10859.

Solenisia Std. 8598.

Somea Wst. 5239.

Somphocarya Torr. 3230.

Sopulina Juss. 8048.

Spadothea Dumort. 8297.

Spalanzania Dtr. 11921.

Spanante Std. 8765.

Sparangium Ritg. 4399.

Spatoglottis Std. 3888.

Spermacocee Dumort. 6655 .

Spermaxyron Std. 10503c.

Sphacellaria B or y 397.

Sphaenopteris St ernb. 2293.

Sphaeralidia Fee 958 a.

Sphaerocarpus Juss. 4256d.

Sphaerocaryum Nees 2761.

Sphaerodesmus Corda, Men. 188.

Sphaerospora Sweet 3644.

Sphaerostemma R ch b. 9342 .

Sphenopecopteris Sternb. 2293.
Sphenostyles Std. 12325.

Spilophora Wst. 491.

Spirea Pall. 11951.

Spirillus Gay 4311/2.

Spirodiscus Jichw. 423.

Spirogera Dumort. 386.

Spirorrhynchus Wst. 9646.

Spirostyles Dumort. 9114n.

Spyridanthus Wst. 5900.

Staafia Wst. 9134.

Stachyarpheta Juss. 7560.

Stachycrater Turez. 9809/1

Stachyditypus Wst. 7515c.

Stachyocnide Blume 4764/1.

Staphilaea All. 10763.

Staphylium Dumort. 8955.

Staurogalium Std. 6630a.

Stauropteris Griseb. 10607 (c).

Steerbeckia Dumort. 1608.

Steinschisma Std. 2761.

Sternatospermum Wst. 2994.

Stemmatophysum Std. 8582.

Stenocalyx Berg 11764. 'Turez. 10634/1.

Stenorrhynchos Spreng. 4165.

Stephanium Juss. 9736.

Stereoxylum Presl 9233.

Stigmaphyllum Wst. 10614.

Stigmasphaera Dumort. 1702k.

Stipandra Dtr. 3489.

Stomandra Jack 11594b.

Stramonia J. Bauh., Herm. 7868.

Strangweja Bertol. 3464/1.

Strizolobium Wst. $12305 \mathrm{~b}$.

Strombodurus Willd. 2888.

Stylephora Rchl. 491.

Stylocorina Gärtn. f. 6911 .

Styxis Dtr. 12637.

Suarda Nocca 11760.

Sullivantia Torr. \& Gr. 9183.

Sutherlandia Räusch. 12900/1.

Suzygium P. Browne 11750.

Swenkia Hedw. 7894.

Syalita Rheede 9362 .

syderitis All. 7507.

Syennema Spr. 723.

Sylibum Dumort. 6246.

Symbasiandra Willd. 2733.

Symphitum A 11. 7673.

Symphyloma Std. 8920.

symphyogyne Wst. 2096.

Synapisma Std. 10903.

Synclostemon Std. 7428.

Synnotia Dumort. 3644.

Synosma Rafin. 6145.

Syphomeris Std. 10343a . 
Systemon Reg. 11383/1.

Syzigium Std. 11770.

Szeglewia Müll. 10270/1.

Szovitzia Wst. 8961.

Tachytes Std. 9798.

Tala Miq. 4686.

Talictrum All. 9392.

Tamara Roxb. 9706.

Tantalus Nor. 10377.

Taphranthus Wst. 11148.

Tapinia Std. 11252.

Tasalia Rz. \& Pav. 12901/1.

Tasmania Wst. 9352.

Taxodites Sternb. 4540.

Taxotrophis Blume 4719/2.

Teichmeyera Wst. 11813.

Teichostemma Std. 6526.

Telopogon Dtr. 3088.

Tempeltonia Std. 12039.

Terrana Wst. 5422b.

Tertianaria J. Bauh. 7406.

Tesota A. Gr., Müll., 12427.

Tessararthra Ehrenb. 179.

Tessarthra Kütz. 179.

Tessellina Dumort. 2065.

Tetragamestus Rehb. f. 3939.

T'etrapterigium Dtr. 9632.

T'etrasperma G. Don 10860/1.

Tetrastemum Wst. 11680a. 11806.

Tetrorrhiza Wst. 73990 .

Thallosphaera Dumort. $1702 \mathrm{~m}$.

Thalysia Linn. 2715.

Theleophytum W'st. 4816.

Theligonis Reuss 4781.

Thelythamnus Wst. 5861.

Thiebaudia Wst. 3889 .

Thommasinia Std. 8883 .

Thompsonia Std. 4347.

Thridax Beckm. 5857.

Thrixidium Wst. 5633c.

Thrixis Wst. 5633b.

Thumbergia Dumort. 8087.

Thurnhausera Std. 7368.

Thylaucium Dtr. 9724.

Thyridostachium Wst. 304\%.

Thyselinum Wst. 8880 .

Tigrida Dumort. 3632.

Tilesia Thunb. 12902/1.

Tilocnide Blume $4763 \mathrm{e}$.

Timbuleta Std. 7918.

Tinaea Garz. 2962.

Tinantia I) umort. 3628.

Tinantia Scheidw. 3308 .

Tinnantia Endl. 3308.

Tinus Burm. 8516.

Tiraseckia Sweet 8501a.
Titanephyllum En dl. 632.

Todda-panua Rheede 2653.

Tomex Linn. 7582.

Toninia Wst. 3293.

Tooldia Lehm. 12902/2.

Torrentia Arrab. 6525/3.

Torrulia Std. 4979.

Tournifortia Wight 7648c.

Toxicodendrum Cat. 12683/2.

Tozzettia Parlat. 3411/1.

Trachelospermum Lemr. 7155.

Trachyrhyngium std. 3182.

Trachystella std. 9385.

Tragopyron Wst. $4945 \mathrm{c}$.

Trasya Dtr. 2756.

Trema Lour. 4688.

Tresanthera Karst. 6861/1.

Triaden oides Waip. $1045 ?$.

Triaridium Dumort. 7659f.

Tribachia Dumort. 3838.

Triceras Wst. 11254.

Trichila Wist. 10569.

Trichoballia Presl 3174/1.

Tricholea D u mor t. 2115.

Trichoneura Anders. 2!19/1.

Trichopodes B. \& W. 11366b.

Trichopterys Wst. 2915.

Trichorrhiza Lindl. 3959.

Trichosantha std. 2810.

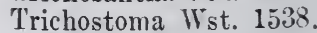

Trifolium Burm. 12218.

Trigonocarpaea Std. 8322.

Trigonostemum Wst. 11062.

Trilipsium std. 11985.

Trillium Mill. 3538.

Trillum Nutt. 3538.

Trimerizia Std. 5197.

Trinax Dtr. 4481.

Trioidia Dtr. 2924.

Triostemum Dtr. 69?0.

Tripethelium Du mort. 962.

Triphocoma Wst. 2195.

Tripterella Rich. 3604 b.

Tristania Poir. 2882.

Tristichis Wst. 2218.

Tristylium Turcz. 10409/1.

Trithomanthe Wist. 3433.

Tritomopteris Wst. 10595 a.

Trommsdorffia Itr. 8332.

Tromsdortfia Std. 4919a.

Tropidocárpon Itr. 9589.

Troximum Std. 6481.

Tulasnea Wight 4647 .

Tulbachia Dtr. 3502.

Tulboghia Wist. 3502.

Turpistra Std. 3574. 
Tylocarpus Wst. 5642 .

Ulek Walp. 5593.

Ulricia Jacq. 7476.

Unannea Std. 7972.

Urgenia Wight $3458 \mathrm{~b}$.

Urocnide Blume 4763f.

Urtica Burm. 4763.

Urticella Miq 4744a.

Uvifera Pluk. 11784/1.

Valcarcella Std. 12500.

Valenzuela Std. 10652.

Vallantia 1)tr. 6627.

Valota Dumort. 3697.

Vanhouttea Lemr. 8411.

Variolaria Sternb. 2621. 2633 (a).

Vasargia Std 5853.

Tasconcellea Planch. 9905.

Tautheria Wst. 3215.

Vellaquezia Wst. 4958.

Veltis Adans. $62336 \mathrm{u}$.

Verniseckia Std. 10480.

Veronicella DC. 9541a.

Vetiveria Virey 3084.

Viborgia Std. 5611.

Vicentia Dtr. 3180 .

Viereya Std. 8713c.

Vigolina Poir. 5854.

Vitidaea Dumort. $8701 \mathrm{k}$.

Vittadenia Std. 5417.

Vittadina Walp. 5417.

Vitteria Peuss 2370.

Vittmania Wight 10829 .

Vittmannia Dtr. 11288.

Vonkhout Wst. 1977.

Vriesea Beer 3770.

Vriesia Gaudich. (3793/1) Del.

Vrolickia Std. 7899.

Walkutfa Std. 12789.

Watzonia Dumort. 3646 .

Weissa Schrank 2235.

Wenlandia Dumort. 9274.

Werniseckia Wst. 10480 .
Whitea Dtr. 8362.

Wiborgia HBK. 5611.

Willemeta Gimel. 4835.

Willugbeia Dumort. 7059.

Withleya Dumort. 7870d.

Woodwillea Walp. 5479.

Wormbia 1)tr. 9363.

Wurmbaea std. 3381.

Xantheum Wight 6527.

Xanthiophoea std, 7437h.

Xanthochysmus Dumort. 10437.

Xanthoreseda G. Don 9747.

Xanthorhaea Dumort. 3524.

Xanthosmia std. 4359.

Xantorrhiza Dtr. 9434.

Xenophontia Arrab. 6526/1.

Xiphizusa Ki ch b. f. 3843.

Xyloaloë Rumph. 5125.

Xylociste Adans. 12792.

Xylocys te P. Browne 12792.

Xylopicron Adans. 9817.

Xylopic rum P. Bromne 93:7.

Xymalobium Std. 7236.

Xyridium std. 3626e.

Yermoloffia Wst. 7521.

Zacintha Arrud. 8535.

Zacynthia Beckm. 6445.

Zalucania std. 5698.

Zauscheria Std. 11497.

Zedoaria Burm. 12907/1.

Zeocrithum Beckin. 3018a.

Zeopyrum I'rin. 3018a.

Zetocapnia Link \& 0.3755.

Zeuxina std. 4171.

Zichia std. 12279.

Ziera Dumort. 11343.

Zizyphora D umort. 7487.

Zoogloea Cohn 318/1.

Zuxine Wight 4171.

Zuzygium A uct. 11750.

Zygosepalon Rchb. f. 3981/1.

Zygosepalum Richb. f. 3981/1. 



